hep-th/0207107

CTP-MIT-3283

\title{
Dynamics with Infinitely Many Time Derivatives and Rolling Tachyons
}

\author{
Nicolas Moeller and Barton Zwiebach \\ Center for Theoretical Physics \\ Massachusetts Institute of Technology, \\ Cambridge, MA 02139, USA \\ E-mail: moeller, zwiebach@mitlns.mit.edu
}

\begin{abstract}
Both in string field theory and in p-adic string theory the equations of motion involve infinite number of time derivatives. We argue that the initial value problem is qualitatively different from that obtained in the limit of many time derivatives in that the space of initial conditions becomes strongly constrained. We calculate the energy-momentum tensor and study in detail time dependent solutions representing tachyons rolling on the p-adic string theory potentials. For even potentials we find surprising small oscillations at the tachyon vacuum. These are not conventional physical states but rather anharmonic oscillations with a nontrivial frequency-amplitude relation. When the potentials are not even, small oscillatory solutions around the bottom must grow in amplitude without a bound. Open string field theory resembles this latter case, the tachyon rolls to the bottom and ever growing oscillations ensue. We discuss the significance of these results for the issues of emerging closed strings and tachyon matter.
\end{abstract}




\section{Contents}

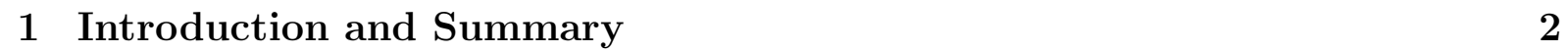

\begin{tabular}{|lll}
2 & Convolution and Initial Value Problem & 7
\end{tabular}

2.1 Convolution form of the p-adic equation of motion . . . . . . . . . . . . . 7

2.2 Convolution constraints on solutions . . . . . . . . . . . . . . . . . . . . 9

2.3 The fate of the initial value problem . . . . . . . . . . . . . . . . . . . . . 13

3 Energy-Momentum in Higher Derivative Theories 15

3.1 Generalized Noether construction . . . . . . . . . . . . . . . . . . . . . . . 15

3.2 Energy in time dependent solutions . . . . . . . . . . . . . . . . . . . . . . 18

3.3 Calculation of the pressure . . . . . . . . . . . . . . . . . . . . . . . . . . . 20

4 Rolling Down the Unstable p-adic Vacuum $\quad 21$

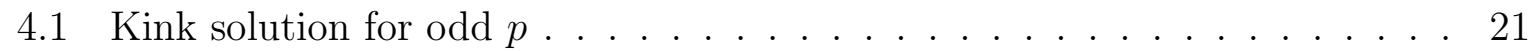

$4.2 \quad$ Rolling tachyons for p-adic strings with even $p$. . . . . . . . . . . . . . 23

4.2 .1 Qualitative discussion of rolling solution . . . . . . . . . . . . . . 24

4.2 .2 Rolling with ever-growing oscillations . . . . . . . . . . . . . . . . 25

4.2 .3 Testing and exploring the solution . . . . . . . . . . . . . . . . 27

\begin{tabular}{|lll}
5 & Anharmonic Oscillations Around the Vacuum & 29
\end{tabular}

$5.1 \quad$ Series construction and amplitude/frequency relation . . . . . . . . . . . . 30

5.2 Energy of anharmonic oscillations . . . . . . . . . . . . . . . . . . . . . . . 34

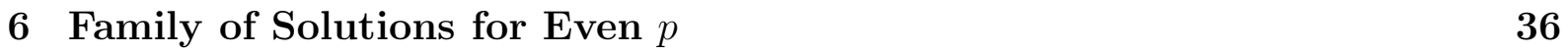

\begin{tabular}{|lll}
7 & Rolling the Tachyon in Open String Field Theory & 38
\end{tabular}

8 Concluding Remarks and Open Questions 4

\section{Introduction and Summary}

Some of the most intriguing and fascinating aspects of string theory center around the role of locality. More precisely, string theory appears to be a consistent theory that is not local in the sense of local quantum field theory. Indeed, the covariant string field theory action governing the dynamics of the infinite set of spacetime fields contains spacetime 
derivatives of all orders. Not only we have spatial derivatives of all orders, as in noncommutative field theory, but we also have time derivatives of all orders, and mixed derivatives of all orders.

The possible difficulties with theories having a high number of time derivatives are familiar. They include possible violations of unitarity and causality, the appearance of spurious solutions, complications setting up an initial value problem, and difficulties quantizing the theory and/or finding a stable Hamiltonian [1]. There is, however, evidence that in the case of string theory the higher derivative structure does not threat the consistency of the theory. The perturbative S-matrix of string theory is unitary. Even though covariant string field theory cannot be quantized using a Hamiltonian, path integral quantization is possible and elegantly done with the use of the Batalin-Vilkovisky formalism [2, 3, 4, 5]. While infinitely many spatial derivatives are possible in non-commutative field theory, infinitely many mixed spatial and time derivatives appear to require string theory for consistency [6, 7]. Finally, the higher derivative structure of string theory is certainly very special - when passing to the light-cone gauge the theory becomes local in light-cone time. Indeed, light-cone string field theory has only first order time derivatives [8].

Motivated by the recent work of Sen on tachyon matter [9, 10, 11] we examine the rolling of the tachyon down its potential. Tachyon matter is a classical open string theory solution where at large times the tachyon $T(t)$ approaches the tachyon vacuum $T=\infty$ with constant velocity $\dot{T}(t)$. This solution represents a pressureless gas and its possible cosmological relevance has been studied in [12]. The evidence for such solutions comes mostly from the conformal field theory approach. Recently, related solutions have been obtained from considerations of boundary string field theory [13, 14]. These actions, truncated to include all powers of first derivatives of fields exhibit similar, though not identical behavior. The purpose of the present paper is to examine tachyon dynamics in the setup of open string field theory [15], where the equations of motion contain infinitely many time derivatives. Most of our work, however, will concentrate in the case of padic string theory [16]. This string theory is essentially a theory of a scalar field with infinitely many spacetime derivatives. Indeed many of the properties of the tachyon field of open string theory are also exhibited by the p-adic string model [17, 18]. One has a tachyon, and a potential with a locally stable minimum where the scalar field has no conventional excitations. Lump-like solutions exist representing p-adic D-branes of various dimensionalities. We will find here interesting phenomena regarding tachyon solutions in p-adic string theory, and we will also see that some solutions in string field theory are quite analogous to p-adic string solutions. None of the solutions we find, should be said, appears to represent tachyon matter.

In order to explain our results we briefly review the p-adic string model. The model 
is defined by an action $S$ for a scalar field. Formulated with an arbitrary spacetime dimensionality, the model has a parameter $p$, which is initially taken to be a prime number. The action, however, makes sense for other values of $p$, and even in the limit $p \rightarrow 1$, where it reduces to the tachyon action of [19] as noted in [20]. In this paper we will take $p$ to be an integer that is greater than or equal to two. The p-adic action is given by

$$
S=\int d^{d} x \mathcal{L}=\frac{1}{g_{p}^{2}} \int d^{d} x\left[-\frac{1}{2} \phi p^{-\frac{1}{2} \square} \phi+\frac{1}{p+1} \phi^{p+1}\right], \quad \frac{1}{g_{p}^{2}} \equiv \frac{1}{g^{2}} \frac{p^{2}}{p-1} .
$$

The infinite number of spacetime derivatives are manifest in the differential operator $p^{-\frac{1}{2} \square}$. Here, we have

$$
\square=-\frac{\partial^{2}}{\partial t^{2}}+\nabla \cdot \nabla
$$

and one defines

$$
p^{-\frac{1}{2} \square}=\exp \left(-\frac{1}{2} \ln p \square\right)=\sum_{n=0}^{\infty}\left(-\frac{1}{2} \ln p\right)^{n} \frac{1}{n !} \square^{n} .
$$

The equation of motion following from the action is rather simple looking:

$$
p^{-\frac{1}{2} \square} \phi=\phi^{p} .
$$

For the case of time dependent but spatially homogeneous solutions - the only kind we will study in this paper - the equation is

$$
p^{\frac{1}{2} \partial_{t}^{2}} \phi=\phi^{p} .
$$

Finally, we note that the scalar potential is given by

$$
V(\phi)=\frac{1}{g_{p}^{2}}\left(\frac{1}{2} \phi^{2}-\frac{1}{p+1} \phi^{p+1}\right) .
$$

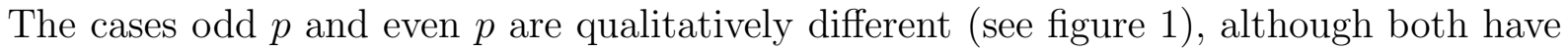
potentials unbounded from below. For odd $p$ the potentials are even in $\phi$, while for even $p$ they fail to be even. In both cases there is a local maximum at $\phi=1$, where the tachyon has $M^{2}=-2$, and a local minimum at $\phi=0$ where the field seems naively to have no dynamics as its mass-squared goes to infinity.

In order to study effectively these theories and their solutions we need expressions for the components of the energy-momentum tensor. For the case of a higher derivative theory we extend the conventional Noether procedure and find an expression for $T_{\alpha}^{\mu}$ as an infinite series involving all numbers of derivatives. These expressions are formally local and can also be used for open string field theory. The energy of a solution does involve 


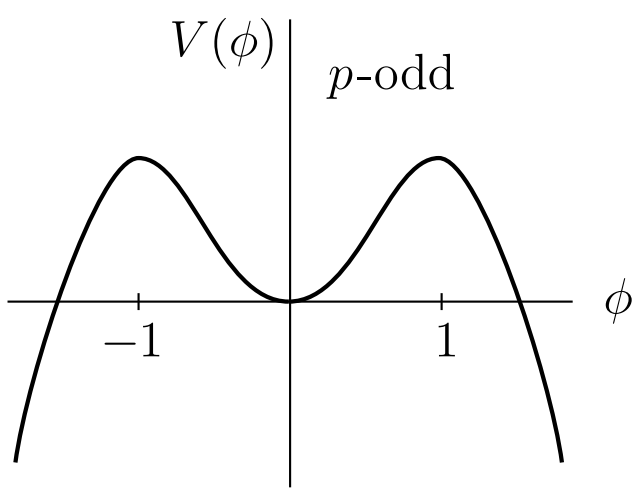

(a)

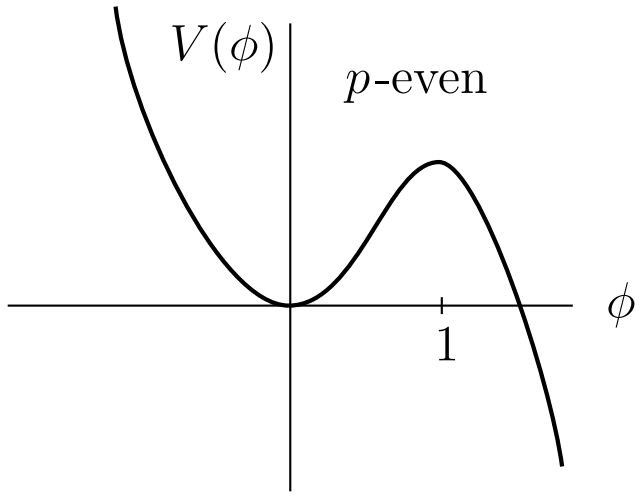

(b)

Figure 1: The p-adic string potentials. For odd $p$ the potential is even and has two unstable maxima. For even $p$ there is only one unstable maximum.

infinite number of derivatives and it is conserved. Its evaluation can sometimes be done in closed form. It is also determined unambiguously from the above Noether method. The Noether calculation of $T_{i}^{i}$, where from we must read the pressure, has improvement ambiguities and we use the metric variation of the covariantized p-adic action to calculate its value.

We also discuss the nature of the initial value problem for equations such as (1.5) containing infinitely many time derivatives. While an equation of motion containing up to $N$ derivatives requires $N$ independent initial conditions, we show that in the case of infinite number of time derivatives the infinite number of "initial conditions" are not independent but are actually subject to (possibly) infinitely many constraints. It may even happen that the space of consistent initial conditions is parametrized by a finite number of parameters. If this were the case we would have a surprising example of a theory with infinitely many time derivatives whose solution space is not altogether different to that following from equations with two time derivatives.

The first time dependent solution in p-adic string theory was found by Brekke et.al. [16]. The equation of motion (1.5) was studied by expressing the differential operator in the left hand side as a convolution of $\phi$ with a gaussian, as will be reviewed in section 2.1. The equation of motion then becomes a non-linear integral equation. For the case of odd $p$ the authors found numerically a kink solution of the integral equation. The solution interpolates in time between the vacua $\phi= \pm 1$. No solution was obtained for even $p$. 
In this paper we study in detail time dependent solutions of p-adic string theory. We use a combination of analytic and numerical methods. In particular we show how to use the convolution approach to constrain solutions and to rule out certain behaviors. Some of the numerical methods are based on considerations of [9, 10] and the recent work [21]. While we have no exact closed forms for any solution, we have analytic expressions valid in certain limits. As the solutions are sometimes quite surprising we try to check them in two ways. We verify they hold numerically both as solutions of the nonlinear integral equation, and after evaluating several derivatives of the solution, we confirm that they directly satisfy (1.5) with the derivatives expanded as in (1.3). This not only increases our confidence on the solutions, but it is of some theoretical significance in that the exponentiated differential operator and the convolution form do not agree when acting on certain kinds of smooth functions.

After confirming the kink solution of [16] to high accuracy and checking it does satisfy both versions of the equation of motion, we turn to finding families of solutions for odd $p$ potentials. We find nontrivial field oscillations around the tachyon vacuum $\phi=0$. These are rather surprising. It is well known that the linearized equations of motion of the tachyon around the vacuum admit no solutions. This is the p-adic version of the familiar statement that there are no conventional open string excitations at the tachyon vacuum [22]. The solutions we find exist because they solve the complete nonlinear equations. The oscillations are completely anharmonic and the basic frequency goes to infinity as the amplitude of the oscillation goes to zero. The solutions are of the form $\phi \simeq A(\cos (\omega t))^{1 / p}$, with $\omega^{2} \sim-\ln (A)$ and provide an explicit example of a non-standard open string excitation capable of carrying energy at the tachyon vacuum. These solutions are obtained by a level analysis of the differential equation and were checked also by the integral form. There are two possible interpretations for these oscillations. In the first one, they would represent oscillating solutions with a nontrivial energy-momentum tensor which would be expected to radiate quite efficiently into closed string modes, thus converting the tachyon energy into closed strings. In the second interpretation these oscillations, suitably quantized would be the closed string excitations themselves. We will discuss both possibilities, but believe the former is more likely to be correct.

In the studies of tachyon matter the tachyon vacuum is at infinity and the tachyon never reaches this point. Moreover, there is no meaning to configurations beyond the tachyon vacuum. On the other hand, in string field theory or in p-adic theory, the tachyon vacuum is at a finite point in the field configuration space and there are field values beyond the tachyon vacuum. In the solutions we obtained the field typically speeds by the tachyon vacuum without difficulty. The case of even $p$, in particular $p=2$, where the potential is cubic resembles open string field theory. For even $p$ we have found no constant amplitude 
oscillations around the tachyon vacuum (but we lack a proof that they cannot exist). For $p=2$ a rolling solution down the unstable maximum at $\phi=1$ is seen to zoom by the tachyon vacuum $\phi=0$ and after an excursion to negative values having potential energies higher than that of the original unstable vacuum, ever growing oscillations occur. In this solution the pressure does not go to zero for large times. The analog for even $p$ of the family of odd $p$ bounded oscillations is a family of solutions that has ever growing oscillations (ever growing oscillations also seem possible for odd $p$ ).

Last but not least we study the rolling problem in the full open string field theory using the analytic continuation of the marginal deformation solution of [23] as advocated in [9]. We find that the tachyon rolls down towards the vacuum, goes beyond it by a large factor, and that a pattern of growing oscillations appears to set it. This surprising result, in many ways, motivated our full analysis of the p-adic string model. Indeed, the results summarized above concerning the p-adic model indicate that rolling in OSFT appear to be remarkably similar to rolling on the $p=2$ potential. This lends credence to the idea that the OSFT solution can be trusted and is not an artifact of some approximation scheme. On the other hand it also suggests that this solution is not tachyon matter.

Some concluding remarks and observations, as well as a detailed list of open questions can be found in the conclusion section.

\section{Convolution and Initial Value Problem}

In this section we begin by considering the convolution form of the p-adic string equation of motion. In this form, the equation is a nonlinear integral equation with a gaussian kernel. The convolution form and the differential form of the equation of motion appear to be equivalent only in the space of real analytic functions.

We then show that the convolution form of the equation of motion is a useful tool that allows qualitative analysis of solutions. We use it to show that there cannot exist solutions of p-adic string theory where the field approaches monotonically the tachyon vacuum for large times. We also use it to find constraints on possible oscillatory solutions and to rule out certain types of lump solutions.

Finally, we discuss the crucial issue of the initial value problem in a theory with infinite number of derivatives.

\subsection{Convolution form of the p-adic equation of motion}

In this subsection we discuss the convolution form of the p-adic string dynamics obtained by [16]. Let us first derive this form. To this end we recall the differential form of the 
equation of motion (1.5):

$$
p^{\frac{1}{2} \partial_{t}^{2}} \phi=\phi^{p} .
$$

As a first step we assume a well defined Fourier transform $\hat{\phi}(k)$ of the p-adic field $\phi(t)$ :

$$
\hat{\phi}(k)=\frac{1}{\sqrt{2 \pi}} \int_{-\infty}^{\infty} e^{i k t} \phi(t) d t,
$$

giving $\phi(t)$ by inverse Fourier transformation:

$$
\phi(t)=\frac{1}{\sqrt{2 \pi}} \int_{-\infty}^{\infty} e^{-i k t} \hat{\phi}(k) d k .
$$

Then, the left hand side of the equation of motion (2.1) can be written as:

$$
\begin{aligned}
p^{\frac{1}{2} \partial_{t}^{2}} \phi(t) & =\frac{1}{\sqrt{2 \pi}} \int_{-\infty}^{\infty} p^{-\frac{1}{2} k^{2}} e^{-i k t} \hat{\phi}(k) d k \\
& =\frac{1}{\sqrt{2 \pi}} \int_{-\infty}^{\infty} d k\left(\frac{1}{\sqrt{2 \pi \ln p}} \int_{-\infty}^{\infty} e^{-\frac{t^{\prime 2}}{2 \ln p}} e^{-i k\left(t-t^{\prime}\right)} d t^{\prime}\right) \hat{\phi}(k) \\
& =\frac{1}{\sqrt{2 \pi \ln p}} \int_{-\infty}^{\infty} e^{-\frac{1}{2 \ln p}\left(t-t^{\prime}\right)^{2}} \phi\left(t^{\prime}\right) d t^{\prime} .
\end{aligned}
$$

Defining the gaussian convolution $\mathcal{C}[\phi]$

$$
\mathcal{C}[\phi](t) \equiv \frac{1}{\sqrt{2 \pi \ln p}} \int_{-\infty}^{\infty} e^{-\frac{1}{2 \ln p}\left(t-t^{\prime}\right)^{2}} \phi\left(t^{\prime}\right) d t^{\prime},
$$

the equation of motion can then be rewritten as

$$
\phi(t)^{p}=\mathcal{C}[\phi](t) .
$$

This is a nonlinear integral equation. Note that the convolution operator is correctly normalized to represent the differential operator: acting on a constant it gives the constant back

$$
\mathcal{C}[a]=a .
$$

Constant solutions $\phi(t)=\phi_{0}$ of the p-adic field equation (2.4) therefore arise when :

$$
\phi_{0}^{p}=\phi_{0} \quad p>1 .
$$

This gives $\phi_{0}=0$ and $\phi_{0}=1$ valid for all $p \geq 2$. For odd $p$ we also have $\phi_{0}=-1$. These solutions all correspond to the tachyon sitting on a critical point of the p-adic string potentials. 
Before we go on and solve either (2.1) or (2.4), we should note that there are smooth functions $\psi(t)$ (i.e. continuous functions with continuous derivatives of all orders) for which the convolution does not represent the action of the differential operator:

$$
p^{-\frac{1}{2} \partial_{t}^{2}} \psi(t) \neq \mathcal{C}[\psi](t)
$$

A simple example is provided by the following smooth function with compact support:

$$
\psi(t)=\left\{\begin{array}{cc}
0, & |t|>b \\
1, & |t|<a .
\end{array}\right.
$$

where $a$ and $b$ are positive numbers such that $b>a$. When $a \leq|t| \leq b, \psi$ interpolates

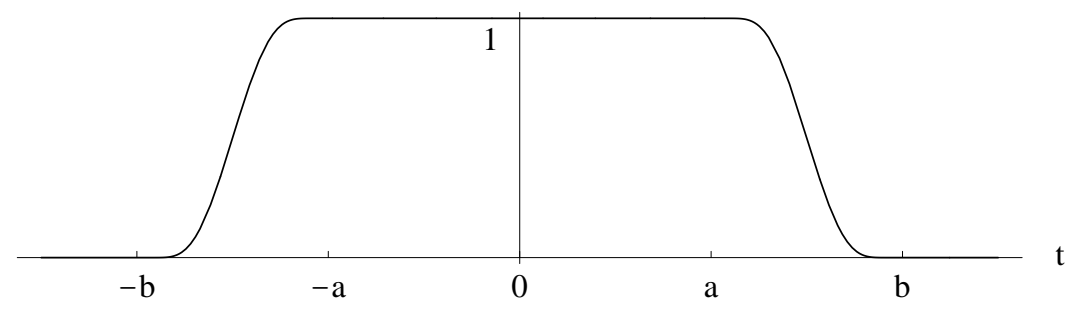

Figure 2: A smooth function that is identically one for $|t|<a$, and identically zero when $|t|>b$. For such a function the differential form of the equation of motion does not coincide with the convolution form.

smoothly between 0 and 1 . Since all the derivatives of $\psi$ are zero at $t=0$, we have $p^{-\frac{1}{2} \square} \psi(0)=\psi(0)=1$; but on the other hand $\mathcal{C}[\psi](0)<1$ since $\mathcal{C}[1]=1$ and $\psi$ is strictly smaller than one when $|t|>a$. Therefore equation (2.2) does not hold for this smooth function. We expect, however, that (2.2) holds for real analytic functions whenever the convolution exists. We have checked many of the solutions obtained in this paper and verified that they solve both forms (2.1) and (2.4) of the equations of motion. This seems to mean that solutions of the p-adic string theory lie on the space of real analytic functions.

\subsection{Convolution constraints on solutions}

General features of a solution to the equation of motion can be gleaned from the properties of the gaussian convolution. We will illustrate this by proving three results that give us insight into the questions we are trying to address. 
The first result rules out the possibility that the tachyon may roll monotonically down from $\phi=1$ reaching the tachyon vacuum $\phi=0$.

Claim 1: There is no solution $\phi(t)$ such that $\phi(t=-\infty)=1$ and $\phi(t=+\infty)=0$ with $\phi(t)$ decreasing monotonically in time.

Proof: Consider a monotonically decreasing function $\phi(t)$ satisfying the above conditions, as illustrated in figure 3. Since the function approaches zero, there is a time $t_{0}$ such that $\phi\left(t_{0}\right)=a$, with $a>0$ and sufficiently small such that

$$
a^{p}<\frac{a}{2}
$$

On the other hand the equation of motion requires

$$
a^{p}=\mathcal{C}[\phi]\left(t_{0}\right)
$$

Consider now the auxiliary function $\psi(t)$ such that $\psi(t)=a$ for $t<t_{0}$, and $\psi(t)=0$ for $t>t_{0}$. It is clear from the monotonicity property that $\phi(t)>\psi(t)$ for all $t$. Moreover, $\psi$ has a very simple gaussian convolution. Using (2.9) we have

$$
a^{p}=\mathcal{C}[\phi]\left(t_{0}\right)>\mathcal{C}[\psi]\left(t_{0}\right)=\frac{a}{2} .
$$

This violates the inequality in (2.8) and proves the claim that no solution exists satisfying the stated conditions.

The second result concerns the possibility of solutions bounded in time. These could be bounded oscillations or otherwise. The ranges of variation are constrained by the following claim:

Claim 2: Consider a solution $\phi(t)$ and constants $a<b$ such that $a \leq \phi(t) \leq b$ for all times $t$. Moreover, assume that the values $a$, and $b$ are actually attained for some specific times. Then $0<b<1$ and $a<0$. For odd $p$ we must also have $a>-1$.

Proof: Let $t_{0}$ denote a time when $\phi\left(t_{0}\right)=b$ and $t_{1}$ denote a time when $\phi\left(t_{1}\right)=a$ (see Figure 4). From the equation of motion we must have

$$
b^{p}=\mathcal{C}[\phi]\left(t_{0}\right), \quad a^{p}=\mathcal{C}[\phi]\left(t_{1}\right) .
$$

Since $\phi\left(t_{1}\right) \leq \phi(t) \leq \phi\left(t_{0}\right)$ we have

$$
b^{p}=\mathcal{C}[\phi]\left(t_{0}\right)<\mathcal{C}\left[\phi\left(t_{0}\right)\right]=b, \quad a^{p}=\mathcal{C}[\phi]\left(t_{1}\right)>\mathcal{C}\left[\phi\left(t_{1}\right)\right]=a,
$$

giving us the inequalities

$$
b^{p}<b, \quad a^{p}>a, \quad b>a,
$$




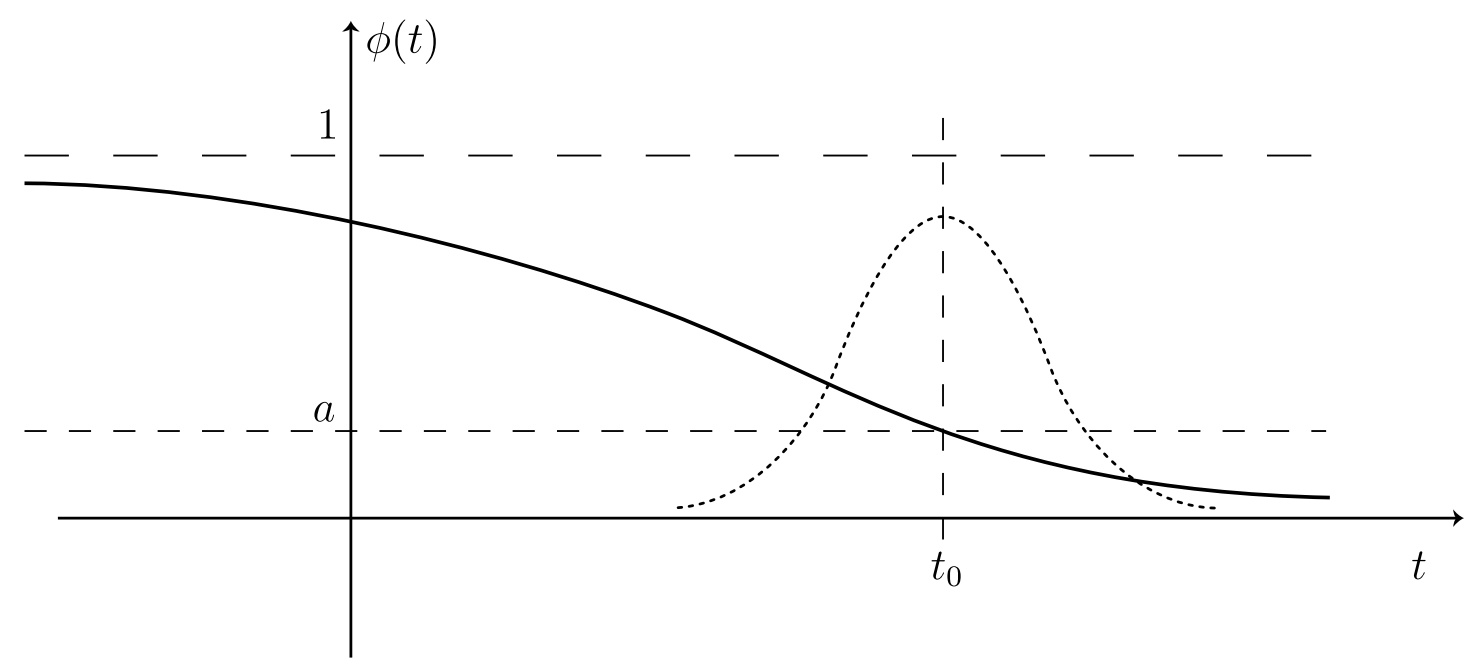

Figure 3: A monotonically decreasing field configuration going from the top of the potential all the way to the tachyon vacuum. Such field configuration cannot satisfy the equation of motion.

where the last inequality is that from the definition of parameters. We rewrite them as

$$
b\left(b^{p-1}-1\right)<0, \quad a\left(a^{p-1}-1\right)>0, \quad b>a .
$$

Note that $p-1$ is a positive integer. Consider the $a$ inequality. If both factors are positive we must have $a>1$. If both factors are negative there are two cases: (i) even $p$ requires $a<0$, and (ii) odd $p$ requires $-1<a<0$. Consider now the $b$ inequality. If $b>0$ then $0<b<1$. If $b<0$ there is no solution for even $p$, and for odd $p$ we see that actually $b<-1$. The inequality $b>a$ allows now selection of the final ranges. Since $b<1$ in all cases, the possibility $a>1$ cannot be realized. Thus $a<0$ always. If $p$ is even then $a<0$ and $0<b<1$. If $p$ is odd then $-1<a<0$. This is not consistent with $b<-1$, and therefore we get again $0<b<1$. This is the statement in the claim.

Our third result will concern the absence of certain lump solutions for the case of even $p$. In order to establish this result we first note that for any bounded function $\phi(t)$ the derivative of its convolution equals the convolution of its derivative:

$$
\frac{d \mathcal{C}[\phi]}{d t}(t)=\mathcal{C}\left[\frac{d \phi}{d t}\right](t)
$$

This result follows by noting that $\frac{d}{d t}$ equals to $\left(-\frac{d}{d t^{\prime}}\right)$ acting on the gaussian appearing 


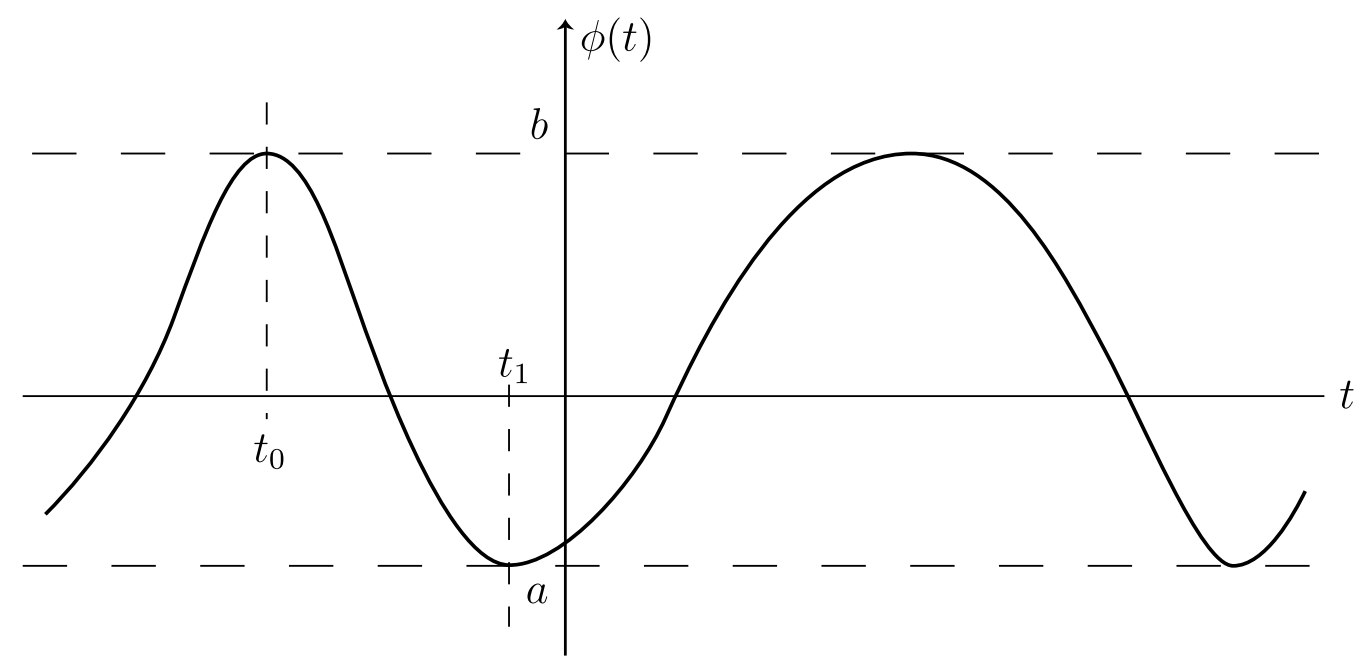

Figure 4: A bounded field configuration attaining a maximum value of $b$ and a minimum value of $a$. The field equation gives constraints on the possible values of $a$ and $b$.

in the convolution (2.3). This derivative can be integrated by parts without picking any boundary contributions and one can let it act on the function $\phi$ thus obtaining the result.

Let us now define a monotonic lump associated to a bounded solution as in claim 2 . This is a solution having $\phi(-\infty)=\phi(\infty)=b$ and $\phi\left(t_{0}\right)=a$ for some unique $t_{0}$. In addition $\phi$ decreases monotonically for $t<t_{0}$ and increases monotonically for $t>t_{0}$. An example of such $\phi$ is shown in figure 5 .

Claim 3: There are no monotonic lump solutions for even $p$.

Proof: Because of claim 2, we have $\phi\left(t_{0}\right)=a<0$. Since $\phi$ has a minimum at $t_{0}$, the convolution $\mathcal{C}[\phi](t)=\phi^{p}$ must have a maximum at $t_{0}$. This requires

$$
\frac{d^{2} \mathcal{C}[\phi]}{d t^{2}}\left(t_{0}\right)<0
$$

We differentiate (2.15) once more to obtain

$$
\frac{d^{2} \mathcal{C}[\phi]}{d t^{2}}\left(t_{0}\right)=\frac{1}{\sqrt{2 \pi \ln p}} \frac{1}{\ln p} \int_{-\infty}^{\infty} e^{-\frac{1}{2 \ln p}\left(t^{\prime}-t_{0}\right)^{2}}\left[\left(t^{\prime}-t_{0}\right) \frac{d \phi\left(t^{\prime}\right)}{d t^{\prime}}\right] d t^{\prime}
$$

From the monotonicity condition we see that the factor in brackets is positive both for $t^{\prime}<t_{0}$ and for $t^{\prime}>t_{0}$. Therefore the above integral is positive, in contradiction with (2.16). This establishes claim 3. 


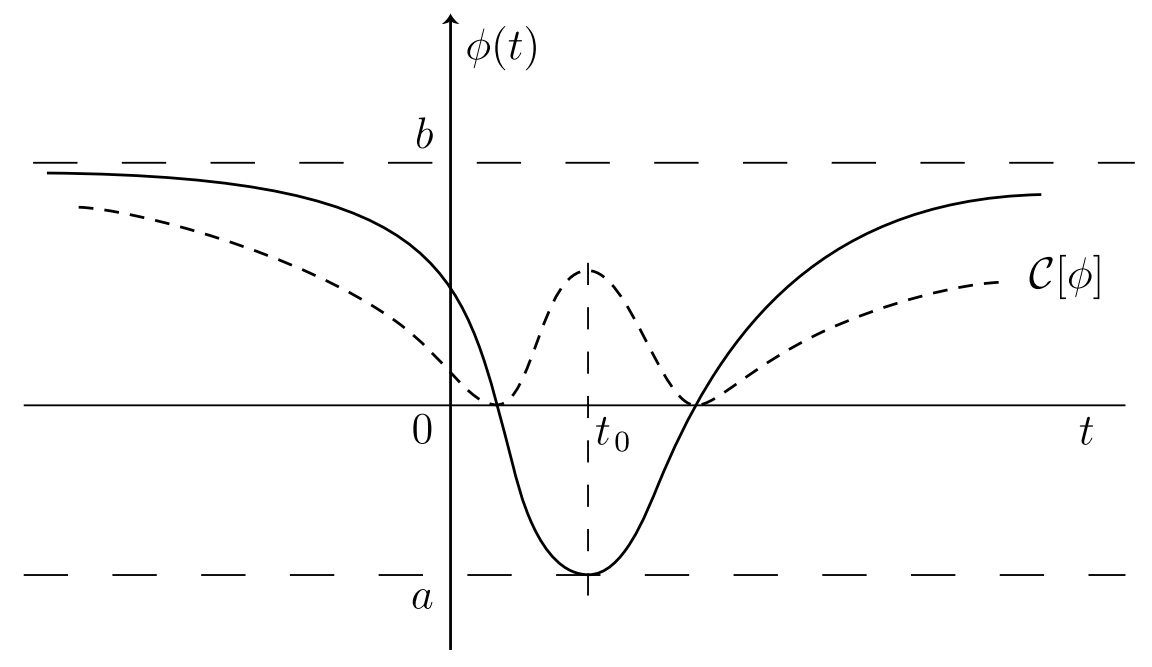

Figure 5: A monotonic lump with endpoint values $b$ and unique minimum value of $a$. This kind of lump solution cannot exist for even $p$. The dashed line represents the gaussian convolution of the field configuration.

\subsection{The fate of the initial value problem}

In this subsection we will make some basic observations about the initial value problem for the equation

$$
p^{\frac{1}{2} \partial_{t}^{2}} \phi=\phi^{p} .
$$

In doing so, we view this as a differential equation for time evolution. This equation has an infinite number of derivatives and it is therefore unclear how to define the initial value problem.

We will argue that an equation as the one above is qualitatively very different from a differential equation having a large but finite number of time derivatives. In solving the initial value problem for a differential equation having time derivatives up to order $N$, one must specify $N$ initial conditions - the values of the field and those of its first $(N-1)$ derivatives at an initial time, for example. Typically, those values are unconstrained, and solutions regular for some finite time exist for any choice of initial conditions.

In an equation such as that in (2.18) one would be led to believe that one can specify an infinite number of independent initial conditions. More precisely, an infinite number minus one - that is, if we specify all derivatives of $\phi$ at zero time, we could use the equation to read the value of $\phi$ at zero time. But a puzzle arises - if the function and all of its derivatives are specified at zero time, the natural assumption of analyticity would 
imply that the function is known and can be reconstructed from its Taylor expansion. It seems the differential equation is not needed anymore!

We will see below that equation (2.18) actually imposes an intricate set of conditions for the initial conditions! We believe the analyticity assumption, and thus think that the evolution is determined fully by the values of the function and all of its time derivatives at the initial time - nevertheless finding consistent initial conditions is now the problem. In the system with infinite number of time derivatives the problem is not that of evolving an initial value configuration, but rather that of constructing a consistent initial value configuration.

In an equation with up to $N$ derivatives, and with $N$ initial conditions, taking further derivatives of the equation typically does not yield constraints for the chosen initial values. The resulting equations simply determine the higher order derivatives at the initial time. On the other hand for equation (2.18) taking arbitrary numbers of time derivatives yields new constraints that must be satisfied by the infinite set of initial conditions. This is what makes the problem different.

To show that there are indeed many constraints implied in (2.18) consider the following example. Let the first derivative of $\phi$ be non-vanishing at zero time, but all higher derivatives vanish:

$$
\phi^{\prime}(0)=a \neq 0, \quad \phi^{\prime \prime}(0)=\phi^{\prime \prime \prime}(0)=\ldots=0 .
$$

Consider now equation (2.18) for $p=2$. Consistent with the above initial conditions we find

$$
p^{\frac{1}{2} \partial_{t}^{2}} \phi(0)=\phi(0)=\phi^{2}(0)
$$

Consider now choosing $\phi(0)=1$ to satisfy this constraint. Since we now have the field and all of its derivatives specified at zero time, the (analytic) field is thus determined to be $\phi=1+a t$, and this does not solve equation (2.18)! What went wrong? We did not have a consistent set of initial conditions. Indeed, taking two derivatives of equation (2.18) we have

$$
p^{\frac{1}{2} \partial_{t}^{2}} \partial_{t}^{2} \phi(0)=2 \phi^{\prime}(0) \phi^{\prime}(0)+2 \phi(0) \phi^{\prime \prime}(0),
$$

which at zero time gives a new constraint on the initial conditions

$$
0=2 a^{2}
$$

This constraint indicates that we should not have expected a solution for nonzero $a$. It also shows that derivatives of the equation of motion yields new constraints on the initial values.

It does not seem altogether unreasonable to expect that equation (2.18) together with all of its derivatives impose an infinite number of consistency conditions on the initial 
values. Could it be that that consistent initial values are parametrized by a finite number of parameters? More exploration is necessary to answer this question.

\section{Energy-Momentum in Higher Derivative Theories}

Both p-adic string theory and string field theory have actions where fields appear with infinitely many derivatives, both spatial and temporal. It is also the case that the action of p-adic string theory has no explicit dependence on the spacetime coordinates. The same occurs for open string field theory in many important backgrounds. It therefore follows that we expect to be able to define a conserved energy-momentum tensor through a generalized Noether procedure. Since the Noether procedure does not yield automatically a symmetric energy momentum tensor, we do not necessarily obtain the energy-momentum tensor that acts as a source of the gravitational field. This causes no complication for the case of the energy, which is unambiguous. But there are total derivative ambiguities in identifying the pressure from the Noether construction of $\left(-T_{i}^{i}\right)$. Therefore pressure is calculated by doing a metric variation in the relativistic covariantization of the p-adic action. We focus on the values of energy and pressure in the case of time dependent but space independent backgrounds, and then specialize further to the p-adic string model.

\subsection{Generalized Noether construction}

The situation in case is described by an action $S$ of the type

$$
S=\int d^{D} x d t \mathcal{L}\left(\phi, \partial_{\nu_{1}} \phi, \partial_{\nu_{1}} \partial_{\nu_{2}} \phi, \partial_{\nu_{1}} \partial_{\nu_{2}} \partial_{\nu_{3}} \phi, \cdots\right),
$$

where the dots denote the dependence on fields acted upon by an ever increasing number of derivatives. The symbol $\phi$ in the above action may represent a collection of fields, but for notational simplicity we will not include an extra index. We emphasize that this collection of fields can include, in addition to scalars, fields of arbitrary tensor type. This is indeed required for the results to be useful in string field theory. All the results below are trivially extended when $\phi$ is a collection of fields.

For a Lagrangian without explicit coordinate dependence the transformation

$$
\delta \phi=\epsilon^{\alpha} \partial_{\alpha} \phi
$$

where $\epsilon^{\alpha}$ is a spacetime constant, gives rise to a conserved current. Under this transformation the Lagrangian varies into a total derivative

$$
\delta \mathcal{L}=\epsilon^{\alpha} \partial_{\alpha} \mathcal{L}=\partial_{\alpha}\left(\epsilon^{\alpha} \mathcal{L}\right)
$$


If the lagrangian $\mathcal{L}$ only contained first order derivatives the stress tensor would be given as

$$
T_{\alpha}^{\mu}=-\delta_{\alpha}^{\mu} \mathcal{L}+\frac{\partial \mathcal{L}}{\partial \phi_{\mu}} \phi_{\alpha}
$$

Here we are using the notation

$$
\phi_{\alpha} \equiv \partial_{\alpha} \phi
$$

or more generally, for any object $A$, we define

$$
A_{\alpha_{1} \alpha_{2} \cdots \alpha_{k}} \equiv \partial_{\alpha_{1}} \partial_{\alpha_{2}} \cdots \partial_{\alpha_{k}} A
$$

The familiar result in (3.4) holds for any field $\phi$ of any tensor type under constant space translations any tensor field transforms as indicated in (3.2). What we wish to have now is the generalization of (3.4) for the case when the action contains all possible numbers of derivatives. Our result will be an expression for the stress tensor involving all numbers of derivatives, and reducing to the above if $\mathcal{L}$ only depends on first derivatives.

We begin the derivation of the general formula by writing the equation of motion following from the action in (3.1). One finds

$$
0=\frac{\partial \mathcal{L}}{\partial \phi}-\left(\frac{\partial \mathcal{L}}{\partial \phi_{\mu_{1}}}\right)_{\mu_{1}}+\left(\frac{\partial \mathcal{L}}{\partial \phi_{\mu_{1} \mu_{2}}}\right)_{\mu_{1} \mu_{2}}-\cdots+(-1)^{k}\left(\frac{\partial \mathcal{L}}{\partial \phi_{\mu_{1} \cdots \mu_{k}}}\right)_{\mu_{1} \cdots \mu_{k}}+\cdots
$$

In here the derivatives are defined as

$$
\frac{\partial \phi_{\mu_{1} \cdots \mu_{k}}}{\partial \phi_{\nu_{1} \cdots \nu_{k}}}=\delta_{\mu_{1}}^{\nu_{1}} \cdots \delta_{\mu_{k}}^{\nu_{k}}
$$

with no additional symmetrizations. Thus while $\phi_{\mu_{1} \cdots \mu_{k}}$ is by definition symmetric in all indices, $\frac{\partial}{\partial \phi_{\nu_{1} \cdots \nu_{k}}}$ is not. It is also useful to note that

$$
\partial_{\alpha} \mathcal{L}=\frac{\partial \mathcal{L}}{\partial \phi} \phi_{\alpha}+\frac{\partial \mathcal{L}}{\partial \phi_{\nu_{1}}} \phi_{\alpha \nu_{1}}+\frac{\partial \mathcal{L}}{\partial \phi_{\nu_{1} \nu_{2}}} \phi_{\alpha \nu_{1} \nu_{2}}+\cdots+\frac{\partial \mathcal{L}}{\partial \phi_{\nu_{1} \cdots \nu_{k}}} \phi_{\alpha \nu_{1} \cdots \nu_{k}}+\cdots
$$

This equation encodes the lack of explicit coordinate dependence in the lagrangian.

At this stage it is simplest to state the result for the energy-momentum tensor and then confirm it is conserved. We find

$$
T_{\alpha}^{\mu}=-\delta_{\alpha}^{\mu} \mathcal{L}
$$

\footnotetext{
${ }^{1}$ For fields other than scalars the resulting energy-momentum tensor $T_{\mu \alpha}$ may not be symmetric.
} 


$$
\begin{aligned}
& +\frac{\partial \mathcal{L}}{\partial \phi_{\mu}} \phi_{\alpha} \\
& -\left\{\left(\frac{\partial \mathcal{L}}{\partial \phi_{\nu_{1} \mu}}\right)_{\nu_{1}} \phi_{\alpha}-\left(\frac{\partial \mathcal{L}}{\partial \phi_{\mu \nu_{1}}}\right) \phi_{\alpha \nu_{1}}\right\} \\
& +\left\{\left(\frac{\partial \mathcal{L}}{\partial \phi_{\nu_{1} \nu_{2} \mu}}\right)_{\nu_{1} \nu_{2}} \phi_{\alpha}-\left(\frac{\partial \mathcal{L}}{\partial \phi_{\nu_{1} \mu \nu_{2}}}\right)_{\nu_{1}} \phi_{\alpha \nu_{2}}+\left(\frac{\partial \mathcal{L}}{\partial \phi_{\mu \nu_{1} \nu_{2}}}\right) \phi_{\alpha \nu_{1} \nu_{2}}\right\} \\
& \quad \vdots \\
& +(-1)^{k}\left\{\left(\frac{\partial \mathcal{L}}{\partial \phi_{\nu_{1} \nu_{2} \cdots \nu_{k} \mu}}\right)_{\nu_{1} \nu_{2} \cdots \nu_{k}} \phi_{\alpha}-\left(\frac{\partial \mathcal{L}}{\partial \phi_{\nu_{1} \nu_{2} \cdots \mu \nu_{k}}}\right)_{\nu_{1} \nu_{2} \cdots \nu_{k-1}} \phi_{\alpha \nu_{k}}\right. \\
& \left.\cdots+(-1)^{k}\left(\frac{\partial \mathcal{L}}{\partial \phi_{\mu \nu_{1} \nu_{2} \cdots \nu_{k}}}\right) \phi_{\alpha \nu_{1} \nu_{2} \cdots \nu_{k}}\right\}
\end{aligned}
$$

We must now verify that

$$
\partial_{\mu} T_{\alpha}^{\mu}=0,
$$

when we use the equation of motion (3.7) and equation (3.9). The verification is straightforward once we note that each group of terms in between braces has a simple derivative. When taking a $\partial_{\mu}$ on any such group the only contributions are from: $\partial_{\mu}$ acting on the $\mathcal{L}$ derivative in the first term, and, from $\partial_{\mu}$ acting on the field derivative in the last term. All other derivatives cancel each other due to the alternating signs in the set of terms. As a result we find

$$
\begin{aligned}
\partial_{\mu} T_{\alpha}^{\mu}= & -\partial_{\alpha} \mathcal{L} \\
& +\left(\frac{\partial \mathcal{L}}{\partial \phi_{\mu}}\right)_{\mu} \phi_{\alpha}+\left(\frac{\partial \mathcal{L}}{\partial \phi_{\mu}}\right) \phi_{\alpha \mu} \\
& +\left\{-\left(\frac{\partial \mathcal{L}}{\partial \phi_{\nu_{1} \mu}}\right)_{\nu_{1} \mu} \phi_{\alpha}+\left(\frac{\partial \mathcal{L}}{\partial \phi_{\mu \nu_{1}}}\right) \phi_{\alpha \mu \nu_{1}}\right\} \\
& +\left\{\left(\frac{\partial \mathcal{L}}{\partial \phi_{\nu_{1} \nu_{2} \mu}}\right)_{\nu_{1} \nu_{2} \mu} \phi_{\alpha}+\left(\frac{\partial \mathcal{L}}{\partial \phi_{\mu \nu_{1} \nu_{2}}}\right) \phi_{\alpha \mu \nu_{1} \nu_{2}}\right\} \\
& \vdots \\
& +\left\{(-1)^{k}\left(\frac{\partial \mathcal{L}}{\partial \phi_{\nu_{1} \nu_{2} \cdots \nu_{k} \mu}}\right)_{\nu_{1} \nu_{2} \cdots \nu_{k} \mu} \phi_{\alpha}+\left(\frac{\partial \mathcal{L}}{\partial \phi_{\mu \nu_{1} \nu_{2} \cdots \nu_{k}}}\right) \phi_{\alpha \mu \nu_{1} \nu_{2} \cdots \nu_{k}}\right\}
\end{aligned}
$$

Using the equation of motion (3.7) one finds that the terms multiplying $\phi_{\alpha}$ add up to $\frac{\partial \mathcal{L}}{\partial \phi}$. Using (3.9) one then sees that indeed $\partial_{\mu} T_{\alpha}^{\mu}=0$.

As written, the expression for the energy-momentum tensor can be used directly to obtain its form in p-adic string theory. As mentioned before, this result can be readily 
extended to a collection of fields $\phi^{i}$. In this case one simply sums over the various fields by replacing in $(3.10)$ the terms

$$
\frac{\partial \mathcal{L}}{\partial \phi_{\ldots}} \phi_{\ldots} \rightarrow \frac{\partial \mathcal{L}}{\partial \phi_{\ldots}^{i}} \phi_{\ldots}^{i}
$$

With this replacement we can use (3.10) to find the energy-momentum tensor associated to a solution in open string field theory.

\subsection{Energy in time dependent solutions}

The focus in this paper is on solutions that have time dependence but no spatial dependence. For these class of solutions the expression (3.10) for the energy momentum tensor simplifies considerably and all the tensor components can only have time dependence. From the conservation law, and with $i, j$, denoting spatial indices,

$$
0=\partial_{\mu} T_{0}^{\mu}=\partial_{0} T_{0}^{0}+\partial_{i} T_{0}^{i}=\partial_{0} T_{0}^{0}
$$

Since the energy $T_{0}^{0}(t)$ is conserved we do not have ambiguities in its construction - an additive constant ambiguity is fixed by the condition that when the field does not vary in time, the energy must coincide with the potential energy.

For solutions with no spatial dependence we also have that

$$
\phi_{\mu_{1} \cdots i \cdots \mu_{k}}=0
$$

since all space derivatives must vanish. Therefore, the terms involving field derivatives in (3.10) vanish when calculating $T_{i}^{0}$ and $T_{j}^{i}$. We find

$$
T_{i}^{0}=0, \quad T_{j}^{i}=-\delta_{j}^{i} \mathcal{L} .
$$

Note that the conservation law $\partial_{\mu} T_{i}^{\mu}=0$ gives $\partial_{0} T_{i}^{0}+\partial_{j} T_{i}^{j}=0$ or equivalently $\partial_{j} T_{i}^{j}=0$. This conservation does not rule out time dependent improvement terms in $T_{i}^{j}$. Therefore the identification of the pressure as $p(t)=-T_{11}=-T_{22}=\cdots=+\mathcal{L}(t)$ is ambiguous. This is also clear from the fact that it is set to equal the Lagrangian, a quantity for which total time derivatives are ambiguous. We will give a computation of the pressure using the methods of general relativity in the following subsection.

Let us now calculate the energy density $T_{0}^{0}$. Denoting, for any quantity $A(t)$

$$
A_{n} \equiv \frac{\partial^{n}}{\partial t^{n}} A
$$


we find from (3.10) that the $k^{\text {th }}$ term in braces becomes

$$
(-1)^{k}\left\{\left(\frac{\partial \mathcal{L}}{\partial \phi_{k+1}}\right)_{k} \phi_{1}-\left(\frac{\partial \mathcal{L}}{\partial \phi_{k+1}}\right)_{k-1} \phi_{2}+\cdots+(-1)^{k}\left(\frac{\partial \mathcal{L}}{\partial \phi_{k+1}}\right)_{0} \phi_{k+1}\right\},
$$

and can be rewritten as

$$
\sum_{m=0}^{k}(-1)^{m}\left(\frac{\partial \mathcal{L}}{\partial \phi_{k+1}}\right)_{m} \phi_{k+1-m}
$$

We then recognize that the complete expression for the energy is simply

$$
E=T_{0}^{0}=-\mathcal{L}+\sum_{k=0}^{\infty} \sum_{m=0}^{k}(-1)^{m}\left(\frac{\partial \mathcal{L}}{\partial \phi_{k+1}}\right)_{m} \phi_{k+1-m}
$$

Shifting the sum over $k$ by one unit we write

$$
E(t)=-\mathcal{L}+\sum_{k=1}^{\infty} \sum_{m=0}^{k-1}(-1)^{m}\left(\frac{\partial \mathcal{L}}{\partial \phi_{k}}\right)_{m} \phi_{k-m}
$$

This is the result we needed. It provides an expansion for the energy in terms of time derivatives of the field. If the lagrangian depends only on finite number of derivatives then the expansion terminates for some finite value of $k$. If the lagrangian has infinite number of time derivatives then the expression for the energy is an infinite series. That series may or may not be possible to sum in closed form. Nevertheless the expression for the energy is formally local - it gives the value of $E(t)$ in terms of derivatives all of which are evaluated at $t$.

We will now evaluate the above expression for the energy in the case of the p-adic string theory (1.1). For any solution $p^{-\frac{1}{2} \square} \phi=\phi^{p}$ of the equation of motion the lagrangian density evaluated at the solution becomes

$$
\mathcal{L}=\frac{1}{g_{p}^{2}} \frac{1}{2} \frac{1-p}{1+p} \phi^{p+1} .
$$

In addition, the derivative $\frac{\partial \mathcal{L}}{\partial \phi_{k}}$ only exists for even $k$, and reads:

$$
\frac{\partial \mathcal{L}}{\partial \phi_{2 \ell}}=-\frac{1}{2 g_{p}^{2}} \phi\left(\frac{1}{2} \ln p\right)^{\ell} \frac{1}{\ell !} .
$$

With this result, the expression for the energy in (3.21) becomes

$$
E(t)=-\mathcal{L}+\frac{1}{g_{p}^{2}} \sum_{\ell=1}^{\infty} \sum_{m=0}^{2 \ell-1}-\frac{1}{2}\left(\frac{1}{2} \ln p\right)^{\ell} \frac{1}{\ell !}(-1)^{m} \phi_{m} \phi_{2 \ell-m},
$$


or, after minor rearrangements,

$$
E(t)=-\mathcal{L}-\frac{1}{2 g_{p}^{2}} \sum_{\ell=1}^{\infty}\left(\frac{1}{2} \ln p\right)^{\ell} \frac{1}{\ell !} \sum_{m=0}^{2 \ell-1}(-1)^{m} \phi_{m} \phi_{2 \ell-m}
$$

This formula will be used to confirm the correctness of certain solutions of p-adic string theory by testing energy conservation. We will also use it in section 5 to compute the energy of oscillatory solutions.

\subsection{Calculation of the pressure}

In this section we use the unambiguous definition of the energy-momentum tensor from general relativity. We make the p-adic model action invariant under coordinate transformations by including a spacetime metric $g_{\alpha \beta}$ and calculate the energy-momentum tensor as

$$
T_{\alpha \beta}=\frac{2}{\sqrt{-g}} \frac{\delta S}{\delta g^{\alpha \beta}} .
$$

The action of the p-adic model, with derivatives expanded and covariantized reads

$$
S=\frac{1}{g_{p}^{2}} \int d^{d} x \sqrt{-g}\left(-\frac{1}{2} \phi^{2}+\frac{1}{p+1} \phi^{p+1}\right)-\frac{1}{2 g_{p}^{2}} \sum_{\ell=1}^{\infty}\left(-\frac{1}{2} \ln p\right)^{\ell} \frac{1}{\ell !} \int d^{d} x \sqrt{-g} \phi \square^{\ell} \phi .
$$

Here the box operators are the covariant ones, and since all of them act on scalars they can be all written as

$$
\square \phi=\frac{1}{\sqrt{-g}} \partial_{\mu}\left(\sqrt{-g} g^{\mu \nu} \partial_{\nu} \phi\right)
$$

The terms that are delicate to vary involve repeated action of box operators:

$$
\begin{aligned}
\int d x \sqrt{-g} \phi \square^{\ell} \phi= & \int d x \phi \partial_{\mu_{1}} \sqrt{-g} g^{\mu_{1} \nu_{1}} \partial_{\nu_{1}} \frac{1}{\sqrt{-g}} \partial_{\mu_{2}} \sqrt{-g} g^{\mu_{2} \nu_{2}} \partial_{\nu_{2}} \cdots \\
& \cdots \frac{1}{\sqrt{-g}} \partial_{\mu_{\ell}} \sqrt{-g} g^{\mu_{\ell} \nu_{\ell}} \partial_{\nu_{\ell}} \phi
\end{aligned}
$$

where all derivatives act to the right. Since we are interested in pressure we will only consider the computation of the components of the energy-momentum tensor $T_{\alpha \beta}$ having spatial indices. Since the solutions we focus on are only time dependent, this means that we need not vary the metric components $g^{\mu_{i} \nu_{i}}$ appearing above for these would yield contributions to the pressure that have explicit spatial derivatives. It therefore suffices to vary the factors of $\sqrt{-g}$, which is done with the help of

$$
\delta \sqrt{-g}=-\frac{1}{2} \sqrt{-g} g_{\alpha \beta} \delta g^{\alpha \beta}, \quad \delta \frac{1}{\sqrt{-g}}=\frac{1}{2} \frac{1}{\sqrt{-g}} g_{\alpha \beta} \delta g^{\alpha \beta} .
$$


When we vary a particular $\sqrt{-g}$ in $(3.29)$ we must integrate by parts all the derivatives to the left of the variation. There are $(2 \ell-1)$ such variations in $(3.29)$, each one giving a different splitting of the derivatives between the two fields. All in all we find (with $i$ not summed) that the pressure is given by

$$
p(t)=-T_{i}^{i}=\frac{1}{g_{p}^{2}}\left(-\frac{1}{2} \phi^{2}+\frac{1}{p+1} \phi^{p+1}\right)+\frac{1}{2 g_{p}^{2}} \sum_{\ell=1}^{\infty}\left(\frac{1}{2} \ln p\right)^{\ell} \frac{1}{\ell !} \sum_{m=1}^{2 \ell-1} \phi_{m} \phi_{2 \ell-m} .
$$

This pressure formula will be used to analyze rolling solutions.

\section{Rolling Down the Unstable p-adic Vacuum}

In this section we construct solutions representing rolling of the tachyon from the unstable vacuum. That is, we want solutions where in the infinite past the tachyon approaches the unstable vacuum. We will obtain a solution for the case of odd $p$ (even potentials) and another very different solution for the case of even $p$.

It was already pointed out in [16] that (2.4) has a kink and an anti-kink solution when $p$ is odd. These are the solutions we are interested in for they start on one maximum at $t \rightarrow-\infty$. As it turns out they end up on the other maximum at $t \rightarrow+\infty$. We will construct such solutions to high accuracy, and verify that they not only solve the convolution form of the field equation, but also the differential form.

The case of even $p$ is much more surprising. Here one could have expected a lump solution, but as we saw in section 2.2 these are not expected to exist. Essentially the difficulties arise because even though the field can go into negative values the convolution, that must equal an even power of the field, cannot. What we find (for $p=2$ ) is

rolling down, an overshooting and then ever-growing oscillations. This is the result of a calculation using an ansatz for the solution in the form of a sum of exponentials. The result is so surprising that we check it in two ways. One by verifying that the solution thus constructed satisfies the convolution form of the field equation, and second, by evaluating the energy and checking that it is conserved for the indicated motion. We also calculate the pressure and find that it does not go to zero. We will see in section that this behavior seems to occur also in OSFT.

\subsection{Kink solution for odd $p$}

Even though the analytical form of the kinks are not known, it is relatively easy to construct them numerically. For example, one can use the iterative procedure where a given field configuration $\phi(t)$ is used to calculate the function $\mathcal{C}[\phi]$. The field equation 
then implies that $(\mathcal{C}[\phi])^{1 / p}$ gives a new (and possibly improved) approximation to $\phi(t)$. Thus we hope for a situation where the iteration

$$
\phi(t) \longrightarrow\left(\frac{1}{\sqrt{2 \pi \ln p}} \int e^{-\frac{1}{2 \ln p}\left(t-t^{\prime}\right)^{2}} \phi\left(t^{\prime}\right) d t^{\prime}\right)^{1 / p},
$$

converges to some definite answer. Note that for odd $p$ there is no complication taking the root - positive numbers are taken to have positive roots, and negative numbers are taken to have negative roots.

Starting with the step function

$$
\phi(t)=\left\{\begin{aligned}
+1, & t<0 \\
0, & t=0 \\
-1, & t>0
\end{aligned}\right.
$$

$\phi(t)$ will converge to the kink solution when we iterate (4.1). Figure 6 shows the kink solutions for $p=3, p=5$ and $p=11$. As can be seen from (2.4) (and as already noted in [16]), near $\phi=0$ the field behaves like $\phi(t) \sim t^{1 / p}$. Also when $p \rightarrow \infty$, the kink tends to a step function. Note that this means the field zooms by the tachyon vacuum crossing it with infinite velocity and spending the least possible amount of time in its vicinity.

Our numerical kink solutions have been constructed from (2.4). As mentioned before, it is important to check that they also satisfy equation (2.1). From equation (1.3) we have that

$$
p^{-\frac{1}{2} \square}=\exp \left(\frac{1}{2} \ln p \partial_{t}^{2}\right)=\sum_{m=0}^{\infty}\left(\frac{\ln p}{2}\right)^{m} \frac{1}{m !} \partial_{t}^{2 m} .
$$

We are thus led to define an approximate version $D^{(2 n)}$ of this differential operator where we include time derivatives of order less than or equal to $2 n$ :

$$
D^{(2 n)} \phi\left(t_{0}\right) \equiv \sum_{m=0}^{n}\left(\frac{\ln p}{2}\right)^{m} \frac{1}{m !}\left(\partial_{t}\right)^{2 m} \phi\left(t_{0}\right) .
$$

In Table 11 we perform a check of the solutions obtained for $p=3$. We choose two values of the field $\phi=0.999,0.99$ to do our check. For these values we compare $\phi^{3}$ to $D^{(2 n)} \phi$, where the derivatives are evaluated using the numerical solution for the $p=3 \mathrm{kink}$. If $\phi\left(t_{0}\right)$ satisfies (2.1), then $D^{(2 n)} \phi\left(t_{0}\right)$ should converge to $\phi^{3}\left(t_{0}\right)$ when $n \rightarrow \infty$. In the first case, only eight derivatives can be evaluated accurately; in the second case, we can trust only six derivatives. In both cases, this suffices to see a relatively good agreement between $D^{(2 n)} \phi\left(t_{0}\right)$ and $\phi^{3}\left(t_{0}\right)$. We thus conclude that the kink obtained by the convolution form of the equation of motion satisfies also the differential form.

If we try to repeat the same check for smaller values of the field, we see that high derivatives have large absolute values, and it would then be necessary to sum a large 


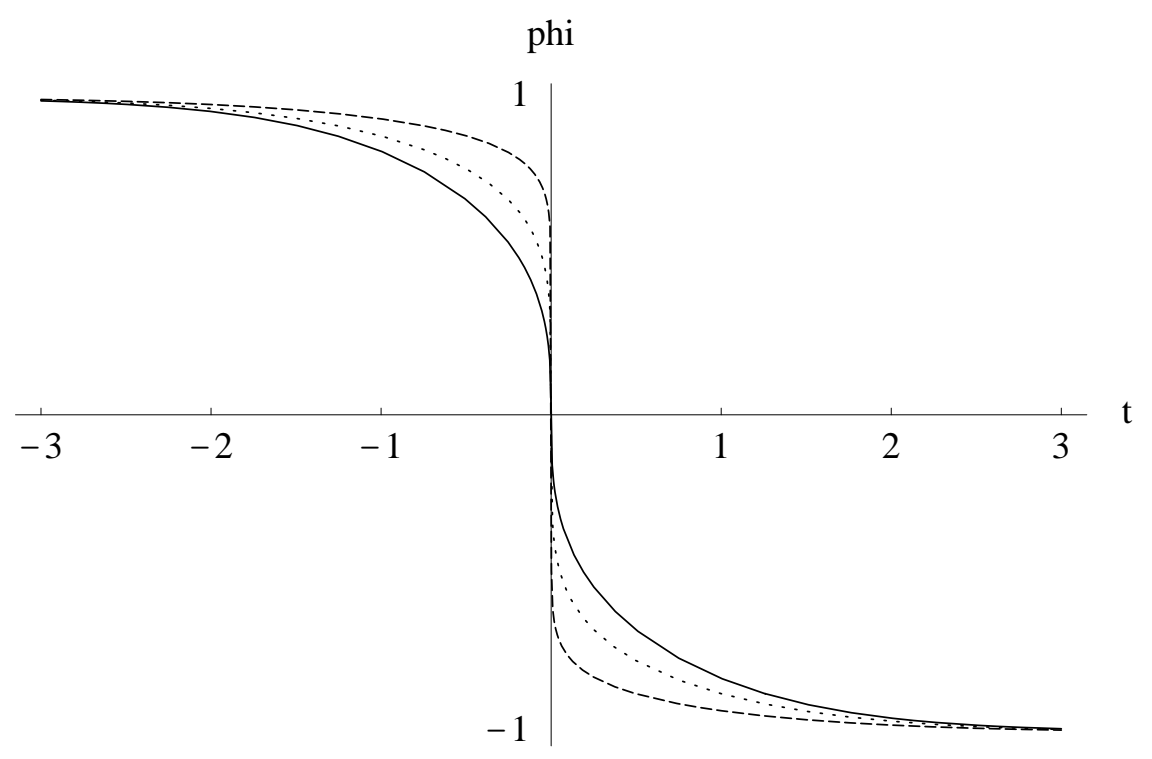

Figure 6: Kink solutions for $p=3$ (solid line), $p=5$ (dotted line), and $p=11$ (dashed line). The horizontal axis is time, and the value of the field $\phi(t)$ interpolates between the unstable vacuum $\phi=1$ in the far past and the unstable vacuum $\phi=-1$ in the far future.

\begin{tabular}{|c|c||c|c|c|c||c|}
\hline$t_{0}$ & $\phi\left(t_{0}\right)$ & $D^{(2)} \phi\left(t_{0}\right)$ & $D^{(4)} \phi\left(t_{0}\right)$ & $D^{(6)} \phi\left(t_{0}\right)$ & $D^{(8)} \phi\left(t_{0}\right)$ & $\phi^{3}\left(t_{0}\right)$ \\
\hline \hline-4.67086 & 0.999 & 0.997905 & 0.997309 & 0.997067 & 0.996974 & 0.997002 \\
\hline \hline-3.04298 & 0.99 & 0.978765 & 0.972588 & 0.971867 & & 0.970299 \\
\hline
\end{tabular}

Table 1: Checking eqn. (2.1) on the numerical kink solution $(p=3)$ computed from (2.4).

number of them to observe convergence. Finer numerical methods would be needed to evaluate enough derivatives. It will become clear in section 5 that the kink is the zerofrequency limit of solutions describing anharmonic oscillations; and this will give us further evidence that the differential form of the equation is indeed satisfied for the kink.

\subsection{Rolling tachyons for p-adic strings with even $p$}

The case of even $p$ is rather different from the case of odd $p$. When $p$ is even the potential has only one maximum $(\phi=1)$ and we cannot have a kink solution. We could expect that the kink gets replaced by a lump-like solution, but as we showed in section 2.2, a typical lump solution does not exist. We will see that if the tachyon rolls down the unstable 
maximum towards the tachyon vacuum it overshoots it and then ever growing oscillations begin. We will first argue qualitatively why such behavior occurs. Then we will calculate the field evolution using a suitable expansion. Finally we will test the solution in two ways.

\subsubsection{Qualitative discussion of rolling solution}

We now imagine the tachyon starts to roll down from $\phi=1$ in the infinite past towards the tachyon vacuum $\phi=0$. Because of claim 1 of section 2.2 we know that the field cannot go asymptotically to the tachyon vacuum for large time. Thus we expect the field to go past $\phi=0$ into negative values, as illustrated in figure 7 where at some time $t_{0}$ we have $\phi\left(t_{0}\right)=0$. As soon as the field turns negative it becomes delicate satisfying the equation of motion, since the convolution must remain positive. This would be very hard to achieve if the field remains negative for a long time. We can therefore expect the field to reach a minimum at some time $t_{1}$ and then go back to zero value at some time $t_{2}$.

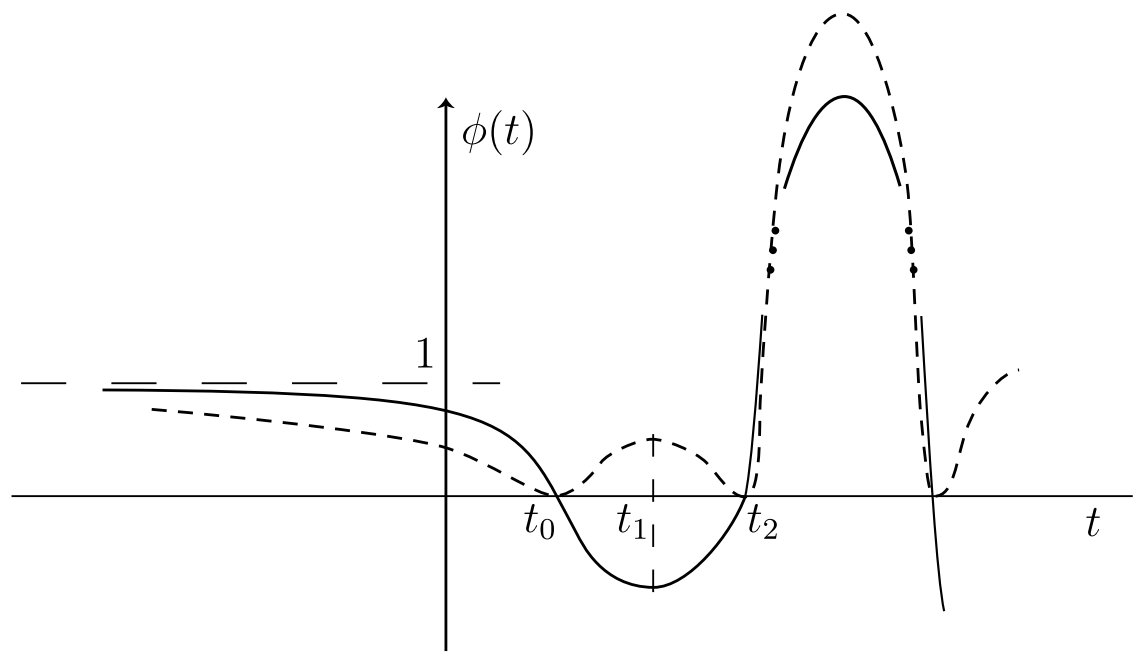

Figure 7: A rolling solution for even $p$ and a consistency analysis based on the value of the convolution $\mathcal{C}[\phi]$ shown in dashed lines.

In figure 7 a dashed line shows the convolution, which must equal $\phi^{p}$. The convolution at $t_{0}$ must be zero, and this is possible since the field is substantially negative to the right of $t_{0}$. But right after $t_{0}$ the convolution must increase. How can that happen when the gaussian peak is moving towards values where $\phi$ is turning more negative ? This can happen if after $t_{2}$ the field $\phi(t)$ is becoming positive very quickly. It must become so large 
and positive that even the fast-decreasing gaussian tail manages to pick up a significant contribution.

Now consider the convolution at $t_{1}$. It can be positive because of the large positive values of the field beyond $t_{2}$. But this convolution must start decreasing to the right of $t_{1}$. It must do so even though the gaussian peak is moving towards values of the field that are large and positive. This can only happen if shortly after $t_{2}$ the field turns negative again and does so extremely fast. All in all we find a pattern of ever growing oscillations. We will now confirm this somewhat qualitative analysis with a computation.

\subsubsection{Rolling with ever-growing oscillations}

We now look for a solution where the tachyon rolls down from its maximum. We shall make the following ansatz

$$
\phi(t)=1-\sum_{n=1}^{\infty} a_{n} e^{\alpha n t},
$$

where $\alpha>0$ is a constant to be determined. Note that we are using "harmonics" of the basic exponential $e^{\alpha t}$. This is sensible due to the structure of the equation we are trying to solve. With $\alpha$ positive, moreover, we guarantee that as $t \rightarrow-\infty$ the field is at the maximum. This kind of expansion has also been discussed in [21].

It is possible to anticipate the value of $\alpha$. It should correspond to $\sqrt{-M^{2}}$, where $M^{2}$ is the value of the mass-squared at the maximum. This can also be derived from the field equation, where we will confirm that $a_{1}$ plays the role of a "marginal" parameter. Since $M^{2}=-2$ at the maximum (for any $p$ ) we must have $\alpha=\sqrt{2}$.

The ansatz (4.5) for the solution is plugged into the left hand side of (2.1) and for $p=2$ we find

$$
p^{\frac{1}{2} \partial_{t}^{2}} \phi(t)=1-\sum_{n=1}^{\infty} a_{n} 2^{\frac{1}{2} \alpha^{2} n^{2}} e^{\alpha n t} .
$$

In addition, the right hand side of (2.1), letting $a_{0} \equiv-1$ takes the form:

$$
(\phi(t))^{2}=\sum_{n=0}^{\infty}\left(\sum_{m=0}^{n} a_{m} a_{n-m}\right) e^{\alpha n t} .
$$

Therefore the equation gives the set of relations

$$
-a_{n} 2^{\frac{1}{2} \alpha^{2} n^{2}}=\sum_{m=0}^{n} a_{m} a_{n-m}, \quad n \geq 1 .
$$

For the case $n=1$ we find the condition:

$$
-a_{1} 2^{\frac{1}{2} \alpha^{2}}=-2 a_{1}
$$


which implies that $\alpha=\sqrt{2}$, and $a_{1}$ is arbitrary. The relations in (4.8) then give

$$
a_{n}\left(2^{n^{2}}-2\right)=-\sum_{m=1}^{n-1} a_{m} a_{n-m} . \quad n \geq 2 .
$$

It is clear from this equation that we can determine the exact values of the coefficients $a_{n}$ iteratively. For example, we readily find

$$
a_{2}=-\frac{a_{1}^{2}}{14} .
$$

It may seem at first sight that we have the freedom to choose $a_{1}$, but a shift in time can be used to rescale it to one (or to minus one if $a_{1}$ is negative, in which case the tachyon rolls towards the unbounded side of the potential). This is possible because $a_{n} \sim a_{1}^{n}$, as can be seen from (4.10) and (4.11), and the structure of the ansatz (4.5).

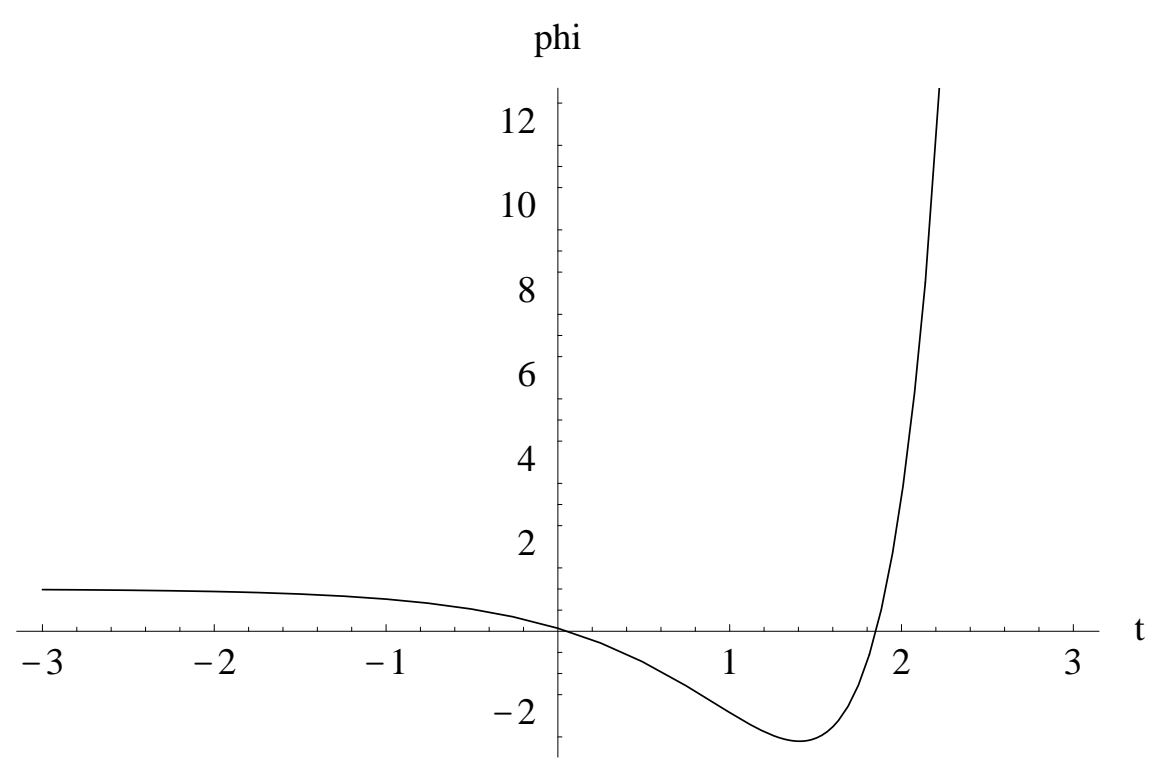

Figure 8: The first oscillation of $\phi(t)$ as function of time. The field oscillates with evergrowing amplitude.

The above iterative procedure can be carried to any desired order to find a solution that is accurate for longer and longer times. Here we show the result for $\phi(t)$ to level five

$$
\begin{aligned}
\phi(t)= & 1-e^{\sqrt{2} t}+\frac{1}{14} e^{2 \sqrt{2} t}-\frac{1}{3570} e^{3 \sqrt{2} t} \\
& +\frac{283}{3275389320} e^{4 \sqrt{2} t}-\frac{7313}{6105767870038200} e^{5 \sqrt{2} t}+\cdots .
\end{aligned}
$$




\begin{tabular}{|c||c|c|c|c|c|c|}
\hline & $t_{0}=0$ & $t_{0}=1$ & $t_{0}=2$ & $t_{0}=3$ & $t_{0}=4$ & $t_{0}=5$ \\
\hline \hline$\phi\left(t_{0}\right)$ & 0.0711485 & -1.92423 & 3.17788 & 184.953 & -424.634 & -196021 \\
\hline$\phi^{2}\left(t_{0}\right)$ & 0.00506212 & 3.70267 & 10.0989 & 34207.7 & 180314 & $3.8424 \cdot 10^{10}$ \\
\hline \hline $\mathcal{C}[\phi]\left(t_{0}\right)$ & 0.00506212 & 3.70267 & 10.0989 & 34207.7 & 180314 & $3.8424 \cdot 10^{10}$ \\
\hline
\end{tabular}

Table 2: Comparison between $\phi^{2}\left(t_{0}\right)$ and $\mathcal{C}[\phi]\left(t_{0}\right)$, where $\phi$ is the solution to level 30 . The agreement is remarkable.

The signs of $a_{n}$ alternate, that will make the field oscillate. In figure 8, we plot the first oscillation. We see that the tachyon starts with the value one, rolls slowly towards the minimum, then overshoots the point $\phi=-\frac{1}{2}$ - the point where the tachyon would turn around in a theory with the same potential and with a canonical kinetic term. Here the field goes down to a value of about $\phi=-2.6$. In doing so the field has climbed a height which is about 55 times that of the original height of the unstable minimum! This can be consistent with energy conservation because in the p-adic string model the kinetic energy can be negative. The field turns around at $\phi=-2.6$, overshooting the maximum and continuing to grow until it reaches a value of about $\phi=593$, a very large value indeed. At this point the potential is very negative and therefore the kinetic energy must be very large and positive. The field then manages to climb up the potential to go to lower values, and continues to oscillate with ever-growing amplitude.

\subsubsection{Testing and exploring the solution}

We want to do two kinds of check on this solution. First, since it was constructed from the derivative form of the equation of motion, we want to make sure that it satisfies the convolution form of the equation of motion. This will also confirm the correctness of the intuition that suggested that convolution requires ever growing oscillations. Then we want to measure the energy of the solution. In particular, we want to confirm the rather unusual property that ever growing oscillations can be compatible with the constancy of the total energy.

In table 2, we compare $\phi^{2}\left(t_{0}\right)$ to the convolution $\mathcal{C}[\phi]\left(t_{0}\right)$ calculated numerically. We have taken $\phi(t)$ to level 30. We see that the agreement holds to at least six digits accuracy. This is impressive given that the field values include very large numbers and therefore are sampling at least two cycles of oscillation.

In the second test we use the energy formula (3.25) to see if the solution satisfies the requirement that the energy is constant. Since the energy formula includes infinite number of derivatives we expect better accuracy as we include more and more terms. 


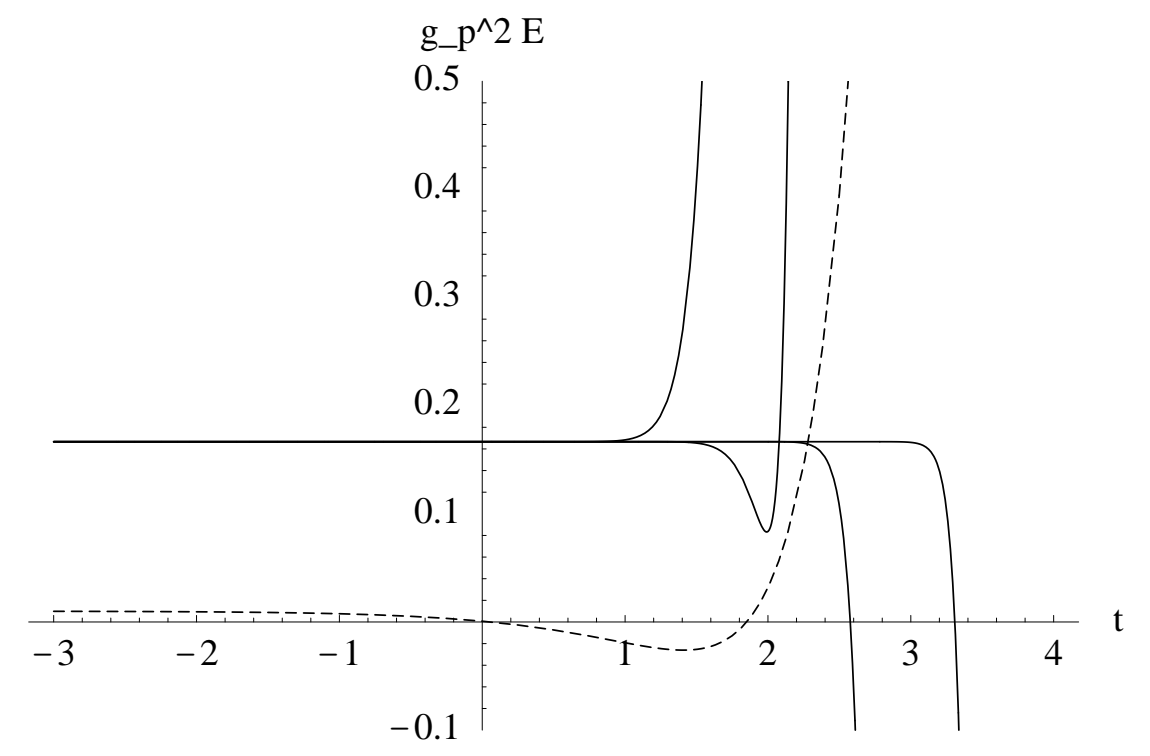

Figure 9: Testing the constancy of the energy $E(t)$ for the rolling solution with ever growing oscillations. This figure shows $g_{p}^{2} E(t)$, calculated from (3.25), using a level 30 solution (4.5). The successively flatter (solid) curves are calculated from (3.25), keeping respectively 40,60, 100, and 150 time derivatives. The dashed curve is $0.01 \phi(t)$ and is just shown as a reference.

This is indeed what we observe in figure 9. We note that including more derivatives in the energy formula makes it constant for longer times (when the higher derivatives of $\phi(t)$ become more important). It is also clear on this graph, that the energy of the rolling solution is $\frac{1}{6 g_{p}^{2}}$, the height of the maximum of the potential.

In figure 10 we show the pressure (multiplied by $g_{p}^{2}$ ) as a function of time. This was calculated using (3.31) and (4.12) evaluating the solution to level 14, and using up to 80 derivatives in the pressure formula. Note that for large negative times the value is negative and equal to $(-1 / 6)$. Shown in dashed lines, for comparison is the pressure including only the first derivative contribution. We see no sign that the pressure is going to zero for large times, therefore no evidence that this solution represents tachyon matter.

It is of interest to compare this solution with similar rolling solutions in ordinary field theory. As noted in 21] the perturbative expansion of a rolling solution in a $\phi^{3}$ theory using the exponentials associated to the unstable maximum breaks down at a finite time. This does not happen with the p-adic string solution. The expansion coefficients $a_{n}$ fall off extremely fast due to the factor of $2^{n^{2}}$ in (4.10). 


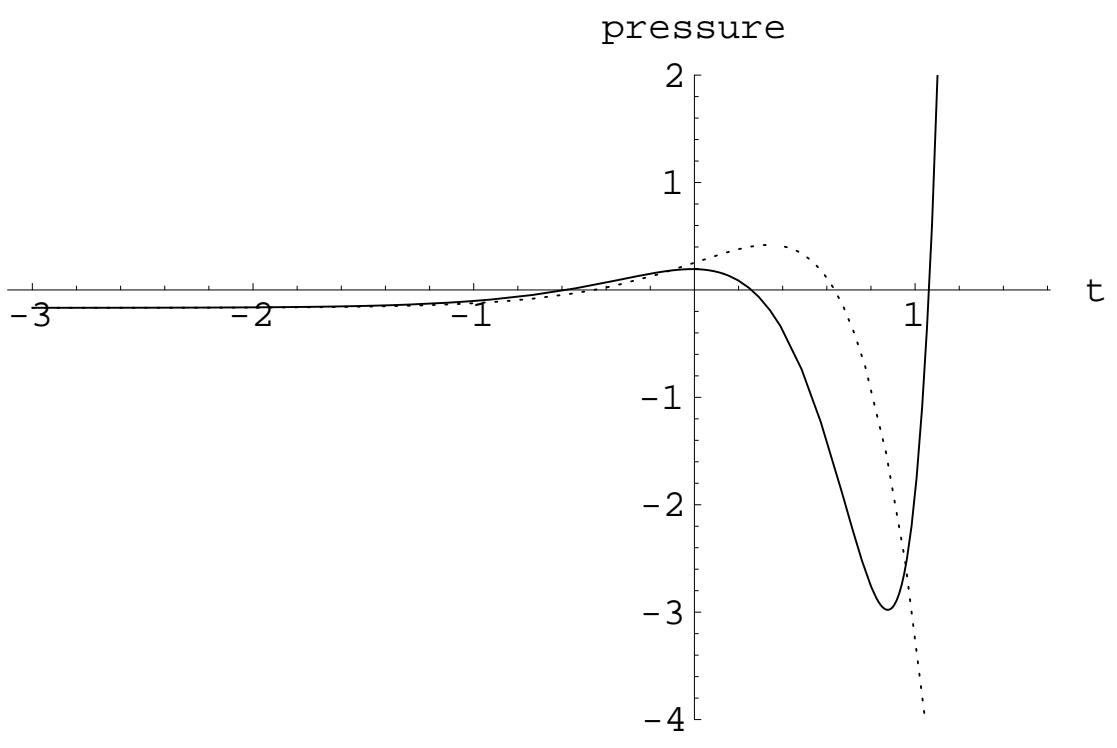

Figure 10: Calculating the pressure of the maximal rolling solution for the $p=2$ potential. Shown in dashed lines is the pressure (multiplied by $g_{p}^{2}$ ) as calculated including only first derivatives in (3.31). In continuous line is the pressure including all derivatives that are relevant in the interval shown.

\section{Anharmonic Oscillations Around the Vacuum}

The p-adic string model, as reviewed in the introduction, satisfies one important property expected from the tachyon. At the minimum, the mass of the field goes to infinity and there are no conventional degrees of freedom. Indeed, for $\phi(t)$ small, the linearized equation of motion is $p^{-\frac{1}{2} \square} \phi=0$, which requires $\square=+\infty$, that is, $m^{2}=\infty$. We therefore have no conventional harmonic oscillations at the bottom of the potential.

It will be seen, however, that while there are no oscillations that solve the linearized equations of motion, there are oscillatory solutions of the nonlinear equations of motion. This is the case when $p$ is odd, and the potential is even. Such oscillations do not appear to exist when $p$ is even.

The oscillations represent a family of solutions - in the limit as the frequency goes to zero they go into the kink solution discussed earlier. In the anharmonic oscillations to be constructed below the amplitude of oscillation is a function of the frequency. As the frequency goes to infinity, the amplitude will go to zero. These oscillations can be found numerically, either by solving the convolution equation by iteration (4.1) starting from a 
periodic $\phi_{0}(t)$, or by a "level" truncation analysis of the differential form of the equation of motion, applied on a Fourier expansion of $\phi$. We will explain this last method in detail, because it will be useful in order to understand the analytic form of $\phi(t)$ in the large frequency limit. It will also allow us to calculate both the amplitude/frequency relation, and the energy of the oscillations in the large $\omega$ limit.

\subsection{Series construction and amplitude/frequency relation}

Let us write a Fourier series for $\phi(t)$ which we imagine oscillates with some amplitude $A$ and period $T$ around the tachyon vacuum $\phi=0$ of an odd $p$ potential. First, we choose the origin of time axis such that $\phi(0)=A$ and $\partial_{t} \phi(0)=0$. Also, since the equation of motion is invariant under $\phi \rightarrow-\phi$, we can demand that $-\phi(t)=\phi\left(t+\frac{T}{2}\right)$, where $T=\frac{2 \pi}{\omega}$, with $\omega$ the fundamental frequency. This implies that $\phi$ can be written in terms of odd modes only

$$
\phi(t)=\sum_{n=0}^{\infty} a_{2 n+1} \cos ((2 n+1) \omega t) .
$$

Since $\omega$ is defined to be the fundamental frequency we assume $a_{1} \neq 0$. It is easily seen that

$$
p^{\frac{1}{2} \partial_{t}^{2}} \phi(t)=\sum_{n=0}^{\infty} a_{2 n+1} p^{-\frac{1}{2} \omega^{2}(2 n+1)^{2}} \cos ((2 n+1) \omega t) .
$$

The right hand side $\phi^{p}(t)$ of the equation of motion can also be expressed as a sum of cosines with odd modes only because $p$ is odd.

Now we want to truncate $\phi(t)$ to level $(2 N+1): \phi(t)=\sum_{n=0}^{N} a_{2 n+1} \cos ((2 n+1) \omega t)$, and for concreteness we will take $p=3$. To level one, $\phi(t)=a_{1} \cos (\omega t)$. Plugging this into the equation of motion gives

$$
p^{-\frac{1}{2} \omega^{2}} a_{1} \cos (\omega t)=\left(a_{1} \cos (\omega t)\right)^{3}=\frac{3}{4} a_{1}^{3} \cos (\omega t)+\frac{1}{4} a_{1}^{3} \cos (3 \omega t),
$$

and, since we work to level one, we keep only the $\cos (\omega t)$ term:

$$
p^{-\frac{1}{2} \omega^{2}} a_{1}=\frac{3}{4} a_{1}^{3}
$$

This equation has the nontrivial solutions

$$
a_{1}= \pm \frac{2}{\sqrt{3}} 3^{-\frac{1}{4} w^{2}}
$$

With $\omega=1$, this is $a_{1}= \pm 0.877383$. Note that at this level $a_{1}$ is the amplitude of oscillation, and it depends nontrivially on the frequency. To level three, $\phi(t)=a_{1} \cos (\omega t)+$ 
$a_{3} \cos (3 \omega t)$, and we have two equations

$$
\begin{aligned}
p^{-\frac{\omega^{2}}{2}} a_{1} & =\frac{3}{4} a_{1}^{3}+\frac{3}{4} a_{1}^{2} a_{3}-\frac{3}{2} a_{1} a_{3}^{2}, \\
p^{-\frac{9 \omega^{2}}{2}} a_{3} & =\frac{1}{4} a_{1}^{3}+\frac{3}{2} a_{1}^{2} a_{3}-\frac{3}{4} a_{3}^{3} .
\end{aligned}
$$

With $\omega=1$, we have, apart from trivial solutions and obvious sign flips,

$$
a_{1}=0.930367, \quad a_{3}=-0.153804 .
$$

With these values the amplitude $A \simeq 0.777$. This is not too far from the value $A \simeq 0.818$ obtained by a rather accurate iterative convolution calculation. In figure 11, we show the solutions to level 1, 3 and 9, compared to the very accurate solution obtained from convolution. At level nine the two different numerical methods give almost the same solution. This means that the oscillations satisfy both the convolution and the differential form of the equations of motion. Since the $\omega \rightarrow 0$ limit of the anharmonic oscillation is the kink, we also expect that the kink is a solution of both equations of motion, as verified to some degree in section 4.1.

We emphasize that in this example we started by choosing a frequency $\omega$ and the numerical solution was then found to be unique (up to obvious time shifts). In particular, the amplitude is given uniquely in terms of $\omega$. In addition, there are solutions for any value of the frequency. This situation is drastically different from the case of classical harmonic oscillations in conventional field theory, where the frequency is given by the mass of the field, but the amplitude is free to take any value.

Although we do not have a closed form solution describing the oscillations, we can derive such closed form in the $\omega \rightarrow \infty$ limit. Indeed, in this limit, the right hand side of (5.2) is dominated by the first term in the sum:

$$
p^{-\frac{1}{2} \square} \phi(t)=a_{1} p^{-\frac{1}{2} \omega^{2}} \cos (\omega t)+\mathcal{O}\left(p^{-\frac{9}{2} \omega^{2}}\right) .
$$

From the equation of motion, we thus have

$$
\phi^{p}(t)=a_{1} p^{-\frac{1}{2} \omega^{2}} \cos (\omega t)+\mathcal{O}\left(p^{-\frac{9}{2} \omega^{2}}\right) .
$$

In the $\omega \rightarrow \infty$, the $\mathcal{O}(.$.$) terms can be neglected and the above equation implies that$

$$
\phi(t) \stackrel{\omega \rightarrow \infty}{\longrightarrow} A[\cos (\omega t)]^{1 / p}
$$

where the amplitude $A$ is related to $a_{1}$ and to $\omega$ as:

$$
A^{p}=a_{1} p^{-\frac{\omega^{2}}{2}}
$$




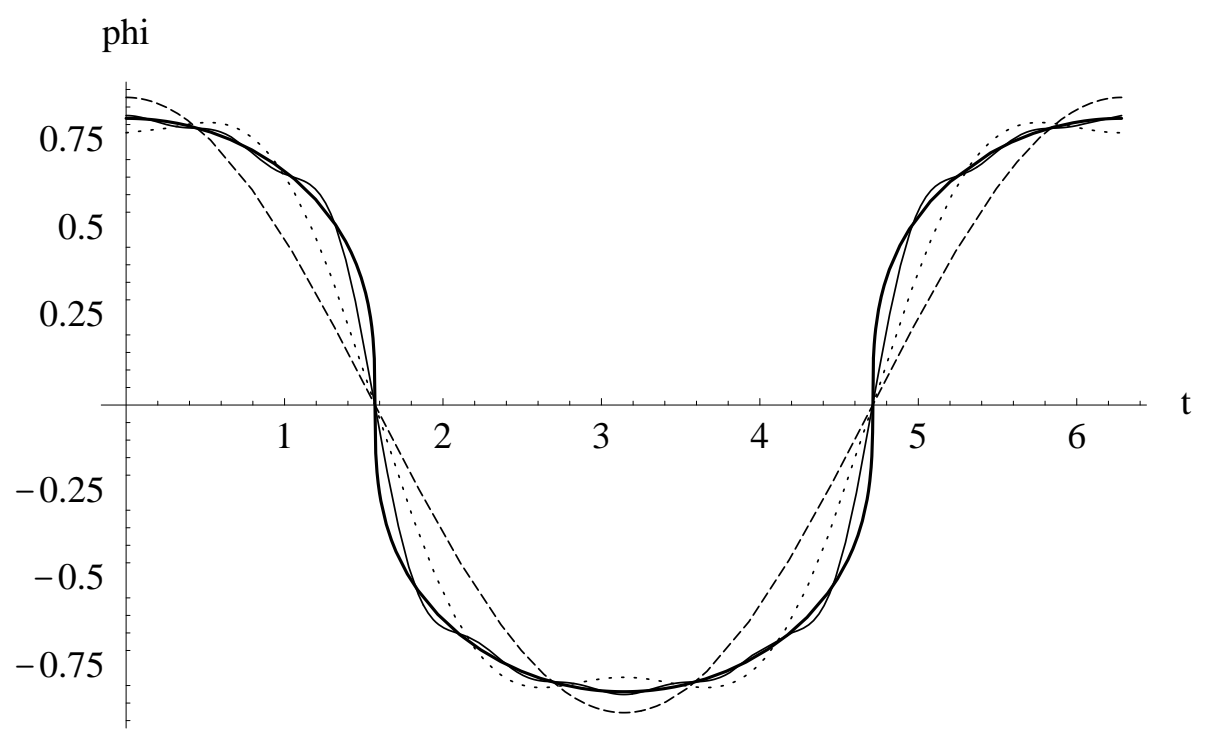

Figure 11: Solutions for the anharmonic oscillation with $p=3$ and $\omega=1$, to levels 1 (dashed line), 3 (dotted line), 9 (thin solid line), and the more accurate solution from the iteration procedure (thick line). The horizontal axis is time. The amplitude is $A=$ 0.817615

Recall that since $p$ is odd, there is no problem in taking the root in (5.11) - for negative arguments the root is defined to be negative. The evaluation of $a_{1}$ is possible from the approximate form of $\phi(t)$. Indeed $a_{1}$ is the first harmonic in the expansion of $[\cos (\omega t)]^{1 / p}$. Using (5.1) we have

$$
A[\cos (\omega t)]^{1 / p}=a_{1} \cos (\omega t)+a_{3} \cos (3 \omega t)+\cdots,
$$

and therefore we can calculate

$$
\begin{aligned}
a_{1}=\frac{\omega}{\pi} \int_{0}^{\frac{2 \pi}{\omega}} \cos (\omega t) \phi(t) d t \stackrel{\omega \rightarrow \infty}{\longrightarrow} \frac{A}{\pi} \int_{0}^{2 \pi}(\cos x)^{1+\frac{1}{p}} d x \\
=A \frac{2 \Gamma\left(1+\frac{1}{2 p}\right)}{\sqrt{\pi} \Gamma\left(\frac{3}{2}+\frac{1}{2 p}\right)} .
\end{aligned}
$$

Now combining (5.12) and (5.14), we find the asymptotic expression for the amplitude:

$$
A^{p-1} \stackrel{\omega \rightarrow \infty}{\longrightarrow} \frac{2 \Gamma\left(1+\frac{1}{2 p}\right)}{\sqrt{\pi} \Gamma\left(\frac{3}{2}+\frac{1}{2 p}\right)} p^{-\frac{\omega^{2}}{2}} .
$$




\begin{tabular}{|c||c|c|c|c|c|}
\hline & $\omega=0.6$ & $\omega=0.8$ & $\omega=1$ & $\omega=1.5$ & $\omega=2$ \\
\hline \hline Numerical amplitude & 0.962253 & 0.899904 & 0.817615 & 0.580459 & 0.358948 \\
\hline \hline Amplitude from (5.15) & 0.975466 & 0.903262 & 0.818225 & 0.58046 & 0.358948 \\
\hline
\end{tabular}

Table 3: Comparison of the amplitudes of the $p=3$ anharmonic oscillations, calculated from the numerical solutions and from the asymptotic formula (5.15).

To illustrate the asymptotic forms we show in figure 12 one period of oscillations for various values of $\omega$ and the asymptotic profile (5.11).
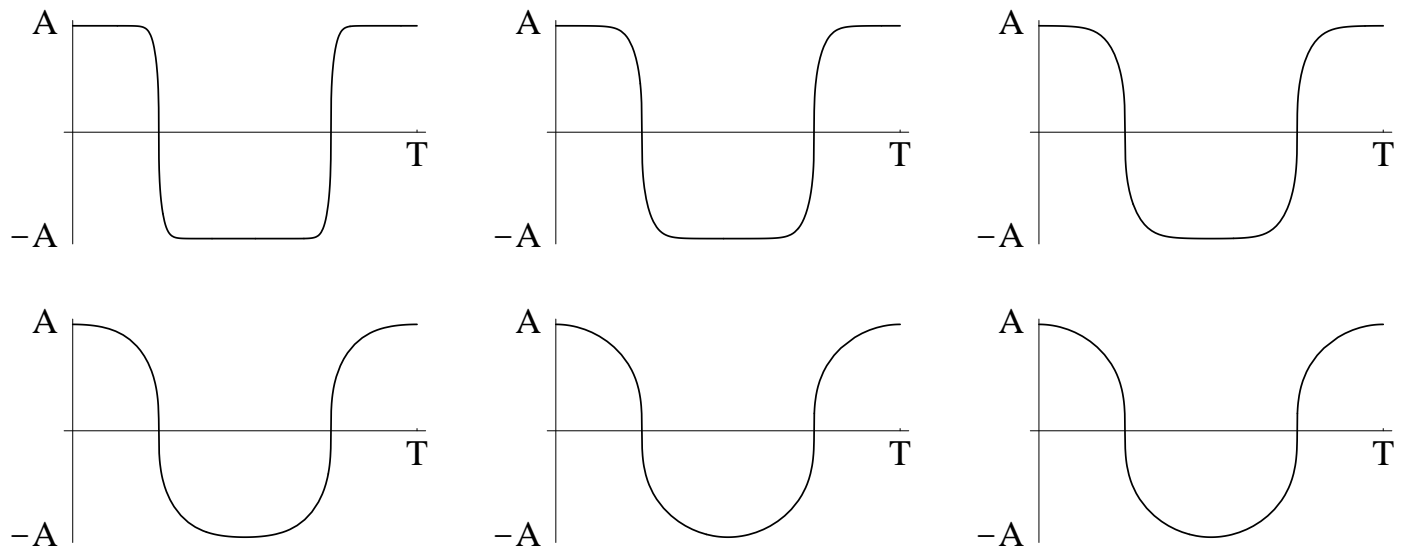

Figure 12: One period $T$ of anharmonic oscillations for $p=3$. On the first row from left to right: $\omega=0.1, \omega=0.2$, and $\omega=0.3$. On the second row from left to right: $\omega=0.5$, $\omega=2$, and the last graph shows the asymptotic profile $(\cos \omega t)^{1 / 3}$. The scale has been adjusted to show all periods as equal.

At last, in table 8, we compare the amplitudes of some $p=3$ numerical solutions (whose absolute accuracy is about $10^{-6}$ ) to the asymptotic amplitudes (5.15). We see that both the profile and the amplitude approach rapidly their asymptotic limits. The asymptotic amplitude is already precise to six digits when $\omega \geq 2$. 


\subsection{Energy of anharmonic oscillations}

It is possible to simplify the formula (3.25) for the energy in the case of anharmonic oscillations. We will eventually find the following asymptotic expression for the energy

$$
E \stackrel{\omega \rightarrow \infty}{\longrightarrow} \frac{h_{p}}{g_{p}^{2}} \omega^{2} A^{p+1}
$$

where $h_{p}$ is a $p$-dependent constant that will be determined in the following calculation.

Let us plug the expansion (5.1) into (3.25). Since the energy is conserved, we can evaluate it at $t=0$; at this particular time, all the odd derivatives of $\phi$ vanish; and the $m$-th derivative, for $m$ even, is

$$
\phi_{m}(0)=\sum_{n=0}^{\infty} a_{2 n+1}(-1)^{\frac{m}{2}}(2 n+1)^{m} \omega^{m} .
$$

Thus (3.25) becomes

$$
\begin{aligned}
E= & -\mathcal{L}(t=0)-\frac{1}{2 g_{p}^{2}} \sum_{\ell=1}^{\infty}\left(\frac{1}{2} \ln p\right)^{\ell} \frac{1}{\ell !} \sum_{\substack{m=0 \\
m \text { even }}}^{2 \ell-2}(-1)^{m} \times \\
& \times \sum_{n=0}^{\infty} a_{2 n+1}(-1)^{\frac{m}{2}}(2 n+1)^{m} \omega^{m} \sum_{k=0}^{\infty} a_{2 k+1}(-1)^{\ell-\frac{m}{2}}(2 k+1)^{2 \ell-m} \omega^{2 \ell-m} .
\end{aligned}
$$

After obvious simplifications, and rearranging the order of summation, we get

$$
E=-\mathcal{L}(t=0)-\frac{1}{2 g_{p}^{2}} \sum_{n, k=0}^{\infty} a_{2 n+1} a_{2 k+1} \sum_{\ell=1}^{\infty}\left(-\frac{\omega^{2}(2 k+1)^{2}}{2} \ln p\right)^{\ell} \frac{1}{\ell !} \sum_{\substack{m=0 \\ m \text { even }}}^{2 \ell-2}\left(\frac{2 n+1}{2 k+1}\right)^{m} .
$$

The last sum is a finite geometric series, it can thus be summed explicitly

$$
\sum_{\substack{m=0 \\
m \text { even }}}^{2 \ell-2}\left(\frac{2 n+1}{2 k+1}\right)^{m}=\left\{\begin{array}{ll}
\ell & , \quad n=k \\
\frac{1-\left(\frac{2 n+1}{2 k+1}\right)^{2 \ell}}{1-\left(\frac{2 n+1}{2 k+1}\right)^{2}}, & n \neq k
\end{array} .\right.
$$

The energy then becomes

$$
\begin{aligned}
E= & -\mathcal{L}(t=0)-\frac{1}{2 g_{p}^{2}} \sum_{n=0}^{\infty} a_{2 n+1}^{2}\left(-\frac{\omega^{2}(2 n+1)^{2}}{2} \ln p\right) \sum_{\ell=0}^{\infty}\left(-\frac{\omega^{2}(2 n+1)^{2}}{2} \ln p\right)^{\ell} \frac{1}{\ell !}- \\
& -\frac{1}{2 g_{p}^{2}} \sum_{\substack{n, k=0 \\
n \neq k}}^{\infty} a_{2 n+1} a_{2 k+1} \frac{1}{1-\left(\frac{2 n+1}{2 k+1}\right)^{2}} \sum_{\ell=1}^{\infty}\left(-\frac{\omega^{2}}{2} \ln p\right)^{\ell} \frac{1}{\ell !}\left((2 k+1)^{2 \ell}-(2 n+1)^{2 \ell}\right) .
\end{aligned}
$$


We can now do explicitly the $\ell$ sums and they give simply exponentials. And since $\phi$ is a solution of the equation of motion, we have

$$
\mathcal{L}(t=0)=\frac{1}{2 g_{p}^{2}} \frac{1-p}{1+p} A^{p+1}
$$

where $A=\phi(0)$ is the amplitude of the oscillation. The expression for the energy thus simplifies to

$$
\begin{aligned}
E= & \frac{1}{2 g_{p}^{2}} \frac{p-1}{p+1} A^{p+1}+\omega^{2} \frac{\ln p}{4 g_{p}^{2}} \sum_{n=0}^{\infty} a_{2 n+1}^{2}(2 n+1)^{2} p^{-\frac{\omega^{2}(2 n+1)^{2}}{2}}+ \\
& +\frac{1}{2 g_{p}^{2}} \sum_{\substack{n, k=0 \\
n \neq k}}^{\infty} a_{2 n+1} a_{2 k+1} \frac{1}{1-\left(\frac{2 n+1}{2 k+1}\right)^{2}}\left(p^{-\frac{\omega^{2}(2 n+1)^{2}}{2}}-p^{-\frac{\omega^{2}(2 k+1)^{2}}{2}}\right) .
\end{aligned}
$$

We now want to find the asymptotic behavior of (5.18) when $\omega \rightarrow \infty$. This is easily done by noting, from (5.15), that $p^{-\frac{\omega^{2}}{2}} \sim A^{p-1}$, and from (5.13), that $a_{2 n+1}^{2} \sim A^{2}$. With this we see that the leading term in the first sum of (5.18) is the first term, and it is of order $\omega^{2} A^{p+1}$. On the other hand the leading term in the second sum is of order $A^{p+1}$. This is also the order of the first term in the equation. Therefore, in the limit that we are considering, the leading term in the energy is

$$
\frac{1}{g_{p}^{2}} \omega^{2} \frac{\ln p}{4} a_{1}^{2} p^{-\frac{\omega^{2}}{2}}=\frac{1}{g_{p}^{2}} \frac{\ln p}{2 \sqrt{\pi}} \frac{\Gamma\left(1+\frac{1}{2 p}\right)}{\Gamma\left(\frac{3}{2}+\frac{1}{2 p}\right)} \omega^{2} A^{p+1},
$$

where we have used (5.14) and (5.15). In total the final expression is

$$
E=\frac{1}{g_{p}^{2}}\left(\frac{\ln p}{2 \sqrt{\pi}} \frac{\Gamma\left(1+\frac{1}{2 p}\right)}{\Gamma\left(\frac{3}{2}+\frac{1}{2 p}\right)} \omega^{2} A^{p+1}+\mathcal{O}\left(A^{p+1}\right)+\text { higher powers of } A\right),
$$

where the first correction is of order $A^{p+1}$, without an $\omega^{2}$ in front of it. The next terms are higher powers of $A$, and their relative contributions are thus exponentially vanishing when $\omega \rightarrow \infty$. It is straightforward to confirm that the above result would arise by computing the energy using simply $\phi=a_{1} \cos (\omega t)$. Thus in this large frequency approximation the first harmonic carries the leading contribution to the energy.

In harmonic oscillations, the energy goes like $A^{2} \omega^{2}$, where $A$ is the amplitude and $\omega$ is the frequency. The frequency dependence of the energy for the anharmonic oscillations is the same, but the amplitude dependence is different - the amplitude appears with the same power as it appears in the potential. Of course, in the present case the amplitude and frequency are not independent variables. 
In summary, we have found physical, energy-carrying excitations around the tachyon vacuum. If we try to interpret them in the context of string theory, they could correspond to non-conventional open string excitations that may radiate into closed strings. In the Sen conjecture, where the tachyon vacuum is supposed to be the closed string vacuum, physical excitations around the closed string vacuum would naturally be interpreted as closed strings. Could the above anharmonic oscillations represent closed strings? Perhaps, but there are a few complications. First, these solutions do not have analogs for even $p$ potentials where any oscillation must eventually grow without limit. In some ways the even $p$ case seems more closely related to bosonic string theory. In addition, we have calculated what would be the analog of classical open string solutions. The closed string states would correspond to the quantization of the above oscillations. Since the closed string spectrum should be coupling constant independent, the quantization must somehow cancel out the factor $1 / g_{o}^{2}$ present in the energy of the classical solutions. For other discussion of closed strings and the tachyon vacuum see 24].

\section{Family of Solutions for Even $p$}

We have already found a family of solutions for the case of odd $p$ - the anharmonic oscillations around the tachyon vacuum. In this section we will present a continuous family of solutions for even $p$ that are, in some sense the closest analog to the anharmonic oscillations of the odd $p$ theory. The oscillations, however, will be unbounded. This can happen even in the limit when the energy can be vanishingly small.

In studying the maximal rolling solution for even $p$ in section 4.2, we saw that the "frequency" $\alpha$ in the expansion (4.5) was forced to take the value $\sqrt{2}$. We will now describe a family of solutions labelled by a parameter $\alpha$ such that $0<\alpha<\sqrt{2}$. For simplicity we will consider only the case $p=2$. Our ansatz is therefore

$$
\phi(t)=\sum_{n=0}^{\infty} a_{n} \cosh (n \alpha t) .
$$

A solution of this form has time-reversal symmetry $\phi(-t)=\phi(t)$. And at $t=0$, the tachyon field has value $\phi(0)=\sum_{n=0}^{\infty} a_{n}$. Let us set up the level truncation scheme for this ansatz. For convenience is it useful to rewrite the expansion as

$$
\phi(t)=\frac{1}{2} \sum_{n=-\infty}^{\infty} \bar{a}_{n} e^{n \alpha t},
$$

with

$$
\bar{a}_{-n}=\bar{a}_{n}=a_{n}, \quad \text { for } \quad n \neq 0, \quad \bar{a}_{0}=2 a_{0} .
$$


Again, the left hand side of the equation of motion is simple

$$
p^{\frac{1}{2} \partial_{t}^{2}} \phi(t)=\frac{1}{2} \sum_{n=-\infty}^{\infty} \bar{a}_{n} p^{\frac{1}{2} n^{2} \alpha^{2}} e^{n \alpha t} .
$$

The right hand side of the equation of motion gives:

$$
\phi^{2}(t)=\frac{1}{4} \sum_{n=-\infty}^{\infty}\left(\sum_{m=-\infty}^{\infty} \bar{a}_{m} \bar{a}_{n-m}\right) e^{n \alpha t} .
$$

The last two equations imply that the equation of motion is equivalent to the set of equations:

$$
\bar{a}_{n} p^{\frac{1}{2} n^{2} \alpha^{2}}=\frac{1}{2} \sum_{m=-\infty}^{\infty} \bar{a}_{m} \bar{a}_{n-m} .
$$

The structure of these equations ensures that it suffices to consider $n \geq 0$. Moreover it is clear that given that the sum in the right hand side is infinite one cannot solve these equations exactly. A level expansion, of course, is possible. To work at level $N$ means to keep all equations above with $0 \leq n \leq N$ and to keep all $\bar{a}_{q}$ with $|q| \leq N$. Finally, let us note that given a solution with expansion coefficients $a_{n}$ we get another solution by letting

$$
a_{n} \rightarrow(-1)^{n} a_{n}
$$

To level zero the equation of motion is $a_{0}=a_{0}^{2}$, and has for solution a tachyon sitting either at the maximum or at the minimum of the potential. To level one, we have two equations for $a_{0}$ and $a_{1}$

$$
\begin{aligned}
& a_{0}=a_{0}^{2}+\frac{a_{1}^{2}}{2}, \\
& 2^{\frac{1}{2} \alpha^{2}} a_{1}=2 a_{0} a_{1} .
\end{aligned}
$$

Apart from trivial solutions, one finds

$$
\begin{aligned}
& a_{0}=2^{\frac{\alpha^{2}}{2}-1} \\
& a_{1}= \pm 2^{\frac{\alpha^{2}}{4}-\frac{1}{2}} \sqrt{2-2^{\frac{\alpha^{2}}{2}}} .
\end{aligned}
$$

Some interesting features can already be seen to this level. First of all, note that we have two branches, indexed by the sign of $a_{1}$, and as could be anticipated by (6.26). If $a_{1}$ is positive, a higher level analysis indicates that so will be all higher coefficients $a_{n}$, and the solution diverges to positive field values without oscillating. When $a_{1}$ is negative, the signs of the $a_{n}$ will alternate, making the solution oscillate with ever-increasing amplitude. 


\begin{tabular}{|c||c|c|c|c|c|c|}
\hline$\alpha$ & 1.41 & 1.4 & 1.3 & 1.2 & 1 & 0.8 \\
\hline \hline$g_{p}^{2} E$ & 0.16082 & 0.147657 & 0.0592576 & 0.0205954 & 0.00114305 & $6.59996 \cdot 10^{-6}$ \\
\hline
\end{tabular}

Table 4: Energy of the solution for some values of the parameter $\alpha$. When $\alpha$ is close to $\sqrt{2}$, we have $g_{p}^{2} E \approx \frac{1}{6}$. And as $\alpha$ decreases, the energy goes to zero very fast.

It is also clear from (6.29) that $\alpha$ has to be smaller than $\sqrt{2}$ in order for $a_{1}$ to be real. In this regard, the maximal rolling solution of section 4.2 , having $\alpha=\sqrt{2}$ can be thought as a limit case. It cannot be obtained, however, by taking the $\alpha \rightarrow \sqrt{2}$ limit of (6.20), that would only give a trivial solution.

The values of the field at $t=0$ and at this present level one approximation are (taking $a_{1}$ to be negative)

$$
\lim _{\alpha \rightarrow \sqrt{2}} \phi(0)=1, \quad \lim _{\alpha \rightarrow 0} \phi(0)=\frac{1}{2}-\frac{1}{\sqrt{2}} \approx-0.207 .
$$

Higher level analysis shows that for any $\alpha$ between 0 and $\sqrt{2}$, the solution oscillates without a bound. When $\alpha \rightarrow \sqrt{2}$, we have $\phi(0) \rightarrow 1$, and the energy tends to $\frac{1}{6 g_{p}^{2}}$, the height of the potential at its maximum. When $\alpha \rightarrow 0$ however, $\phi(0) \rightarrow 0$ and the field becomes flatter near $t=0$, in other words one has to wait for longer times before we see it taking large values. This is illustrated in figure 13. Moreover the energy goes to zero very fast as $\alpha$ becomes small. Even though in these solutions the field can spend a long time oscillating around the tachyon vacuum their energy is necessarily very small, and thus they cannot represent tachyon matter. In table 1 , we show a few values calculated from the formula (3.25) applied on numerical solutions at level 30.

\section{Rolling the Tachyon in Open String Field Theory}

In this section we study the rolling of the tachyon in OSFT. There are at least two ways we could do this analysis. In the first one, we would use level expansion to focus on a few low levels, write the equations of motion (containing infinitely many time derivatives) and solve them as in the p-adic string case. A second method was proposed in ref. [9] (section 2 ), and it uses the analytic continuation of the marginal string field theory solution of ref. [23]. This is the method we will follow here to examine the rolling tachyon.

We will consider the solution representing a marginal deformation of a D1 brane stretched along a circle of unit radius. This marginal deformation can be used to interpolate from the D1 into a D0 brane, and it involves tachyon and higher field harmonics of the type $\cos (n X)$, where $X$ is a coordinate along the circle. The analytic continuation 


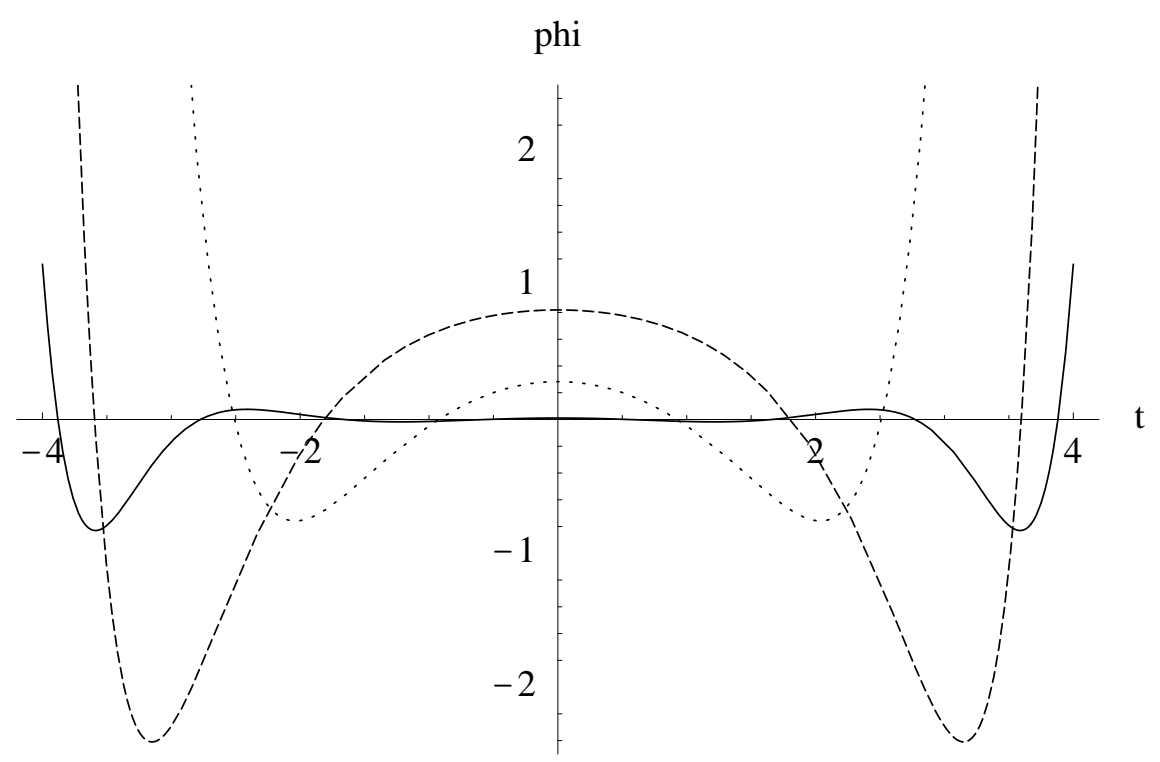

Figure 13: Tachyon profiles as functions of $t$ for $\alpha=1.4$ (dashed line), $\alpha=1.2$ (dotted line), and $\alpha=0.8$ (solid line). The curves become flatter near the origin when $\alpha$ is decreased.

requires replacing $\cos (n X) \rightarrow \cosh (n t)$. We use the solutions of [23], section 3, and let the radius go to one. In addition, we work including fields up to level four and interactions up to level eight, using the action given in Appendix B of [23]. In this way we can include up to the second harmonic of the tachyon. The marginal direction is represented by the first tachyon harmonic $T_{1}$ appearing in the tachyon field expansion

$$
T(t)=T_{0}+T_{1} \cosh (t)+T_{2} \cosh (2 t)+\cdots .
$$

The string field equations are solved by setting a value for $T_{1}$ and solving for the other components of the string field. We take $T_{1}=0.05$ and the equations of motion give

$$
T(t)=0.00162997+0.05 \cosh (t)-0.000189714 \cosh (2 t) \text {. }
$$

It is in fact this result that motivated much of the analysis in the present paper, and a few comments are thus in order. First note that use of the cosh expansion implies that the field satisfies $\dot{T}(0)=0$. The other initial condition could be viewed as giving the value of the tachyon at $t=0$. But certainly that is not the way the equation is solved. Fixing $T_{1}$ one solves for all other harmonics $T_{0}, T_{2}, \cdots$, and all of them together fix the initial value of the tachyon. For $T_{1}=0.05$ we find from the above equation that $T(0)=0.05144$ 


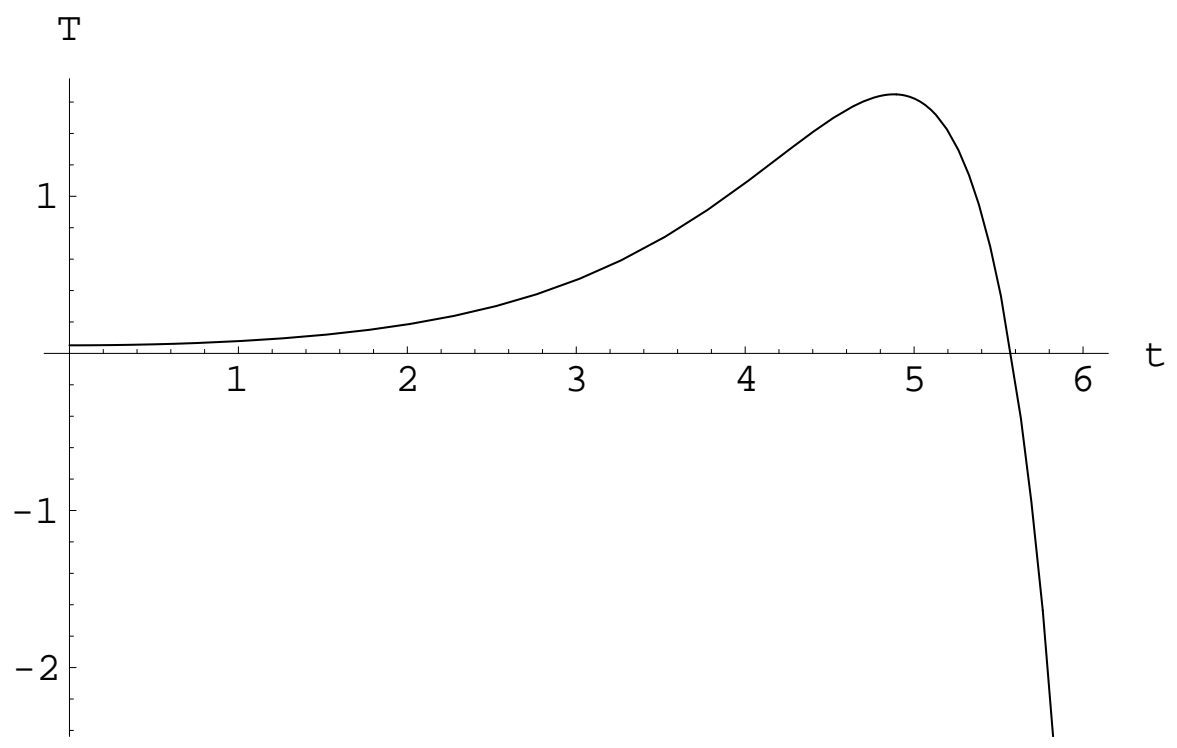

Figure 14: The tachyon profile as a function of time as it rolls down in OSFT. The initial conditions are $T(0)=0.05144$ and $T^{\prime}(0)=0$. The tachyon rolls past the tachyon vacuum $T \simeq 0.55$ and the first turning point is at about $T \simeq 1.65$.

and $T^{\prime}(0)=0$. The first harmonic $\cosh (t)$ reflects the blowing up of the tachyon in the approximation where the potential is quadratic. Note that the second harmonic comes about with negative sign, thus at some time the tachyon stops growing and turns around. We had expected naively that this turning point would be around the tachyon vacuum, thus signaling in this approximation that the tachyon does not cross over. But the result does not support this expectation. In here the tachyon overshoots the tachyon vacuum by a large factor. The maximum is reached for $t \simeq 4.88$ and gives $T=1.649$, which is about three times the value $T \simeq 0.55$ representing the tachyon vacuum. The tachyon as a function of time, is shown in figure 14. We have verified that the turning point $T \simeq 1.65$ is quite stable under small changes of the marginal parameter $T_{1}$, supporting the conclusion that the tachyon generically overshoots the tachyon vacuum before turning around.

The analogy with the $p=2$ maximal rolling solution is actually quite striking. Recall the discussion below eqn. (4.12), where we observed that the p-adic tachyon climbs a height equal to about 55 times the height of the unstable vacuum. We can estimate easily the corresponding quantity in the above OSFT computation. Taking the tachyon potential to be the level zero one $V=-\frac{1}{2} T^{2}+\frac{27 \sqrt{3}}{64} T^{3}$, an overshooting to $T=1.65$ implies that the OSFT tachyon climbs up about 56 times the height of the unstable vacuum! While 
such close agreement is most likely a coincidence, the fact that these quantities are close confirms that the p-adic model seems to capture quite well OSFT features in a simpler context.

What happens next? To answer this we would need the value of $T_{3}$ the third tachyon harmonic. This, however, would require a level $(9,18)$ computation which is a major new task. There are indications, however, that the next coefficient in (7.2) would be positive, and that ever growing oscillations occur, just as in the case of p-adic strings with even $p$.

For the value $T_{1}=0.05$ used above, the next three fields $u, v$ and $w$, all of them at level 2, are:

$$
\begin{aligned}
u(t) & =0.000663831+9.216 \times 10^{-7} \cosh (t), \\
v(t) & =-0.00162516+0.00003999 \cosh (t) \\
w(t) & =0.000301312-6.325 \times 10^{-6} \cosh (t) .
\end{aligned}
$$

With such small values for the first harmonic, these fields do not appear to change significantly the physics of the tachyon. This was also the case in the original marginal deformation problem, where level expansion was seen to operate quite well. In this level $(4,8)$ solution there are additional fields at level three and at level four. We will not write here their values.

We will not attempt here a computation of the pressure in this OSFT solution, but such computation would be of interest. With the behavior of the solution strikingly similar to that of the $\mathrm{p}$-adic string solution for $p=2$, we see no reason to expect that the pressure goes to zero asymptotically, but there could be surprises. A computation would settle this important issue.

\section{Concluding Remarks and Open Questions}

In this paper we have studied tachyon dynamics in string field theory and in p-adic string theory facing up to the novelties due to the infinite number of time derivatives in the field equations.

Several classes of rolling solutions of p-adic string theory have been studied. We have not been exhaustive in showing all of them nor in trying to show that we have a complete set of solutions. For example, there are non-oscillatory diverging solutions for $p=2$ related by the sign change in (6.26) to the oscillatory solutions. In addition there are similar solutions for $p=3$ (and possibly all odd $p$ ). These solutions also show ever growing oscillations, and can be modified by the sign change in (6.26) and by an overall change of sign. There are also additional solutions (both for even and odd $p$ ) arising 
from the analytic continuation of the familiar lump solutions of p-adic string theory. At present, for any value of the energy we know of a finite number of solutions.

In general terms our analysis has revealed a few surprises:

- The solution space of the equations of motions appears to consist of real analytic (smooth) functions. Solutions appear to be in one-to-one correspondence with consistent initial values for the field and all of its derivatives. The dynamical equation imposes a possibly infinite number of conditions on the initial values and thus restricts considerably the solution space. This remarkable feature of the equation of motion was not anticipated by the consideration of systems with finite number of time derivatives.

- There are energy-carrying solutions of the field equations around the tachyon vacuum. These bounded but anharmonic oscillations shown to exist in the case of p-adic strings with even potentials, represent solutions of the non-linear equations not anticipated by the linearized (cohomology-like) problem that admits no solutions.

- There exist oscillatory solutions with ever-growing amplitudes and constant energy. In fact, such solutions exist even for energies that approach zero. These solutions exhibit behaviors not seen in lagrangians quadratic in first time derivatives where the kinetic energy is positive definite. In the systems considered here the kinetic energy can be negative and thus one can see the tachyon move to higher and higher heights on the tachyon potential while conserving the total energy.

At the technical level we have calculated the energy-momentum tensor in higher derivative theories, finding useful expressions for the energy and the pressure in the case of time-dependent solutions. We have also learned how to extract qualitative behavior of the solutions using the convolution form of the field equation. Finally, we have shown how to obtain solutions by numerical methods, and by analytic methods in certain limits.

Interesting open questions remain. We list below a few of them:

- From the physical viewpoint, the most puzzling result has been that none of the rolling solutions obtained here appear to represent tachyon matter. That is, we have not found solutions where with varying values of the energy the pressure goes to zero for long times. The rolling solutions described in this paper would seem to yield oscillating energy-momentum tensors that could effectively convert the D-brane energy into closed string excitations. This had been the conventional wisdom (see, for example [25]) before studies of tachyon matter. Given the exciting possibility that 
a stable form of tachyon matter could have astrophysical consequences it seems of utmost importance to confirm its (theoretical!) existence using string field theory. Since the tachyon vacuum is at a finite and apparently regular value in the field space of string field theory, tachyon matter must be, if present, an exotic solution.

- For the case of even p-adic potentials, the anharmonic excitations around the tachyon vacuum do represent non-conventional type of excitations. The most straightforward interpretation is that they are degrees of freedom that would radiate their energy into closed strings. Could they actually represent closed strings? While preliminary indications do not support this interpretation, further investigation of the physics and interpretation of these solutions are of interest.

- We have seen that there is no conventional initial value problem in the sense that finding consistent initial conditions appears to be the same problem as finding the solution. The space of consistent initial conditions appears to be strongly constrained in the nonlinear equations of motion of p-adic string theory. How many parameters does the space of consistent initial conditions have?

- Our work has given an explicit construction for the energy density of spatially homogeneous solutions in OSFT. The calculation of the pressure was more subtle and was only done for the p-adic string. The OSFT pressure should be computed to test if, as opposed to the case of the p-adic model, the ever growing oscillations could have an asymptotically vanishing pressure. A more geometrical construction would use open/closed string field theory [26], where the energy-momentum tensor would arise from a variation of the closed string field. It may be of interest to give such stringy construction.

- One feature of string field theory is that the infinitely many time derivatives of the covariant formulation turn into first order time derivatives in the light cone formulation. Studies of gauge fixing in string field theory have not revealed how this transformation actually happens. It would therefore be of interest to understand if there is a light-cone gauge p-adic string theory. If the answer is affirmative the passage from covariant to light-cone formulation could be tractable and provide much insight.

- The convolution form of the field equation seems to hide completely the causality properties of the time evolution. It would be of interest to formulate and explore a test of causality in p-adic string theory. 
It is clear that in p-adic string theory and in OSFT we have a rich set of problems and issues that should allow us to improve our understanding of time evolution in string theory. Such understanding seems essential for the development of string theory cosmology.

Acknowledgements. We would like to thank Joe Minahan and Ashoke Sen for many stimulating conversations and discussions throughout this work and critical comments on a draft. N. M. wants to thank Dru Renner for help with the C language. B. Z. would like to acknowledge helpful conversations with M. Headrick, E. Martinec and N. Nekrasov at Cargese 2002. This work was supported in part by DOE contract \#DE-FC02-94ER40818.

\section{References}

[1] D. A. Eliezer and R. P. Woodard, "The Problem Of Nonlocality In String Theory," Nucl. Phys. B 325, 389 (1989). R. P. Woodard, "A canonical formalism for Lagrangians with nonlocality of finite extent," Phys. Rev. A 62, 052105 (2000) arXiv:hep-th/0006207. D. L. Bennett, H. B. Nielsen and R. P. Woodard, "The initial value problem for maximally non-local actions," Phys. Rev. D 57, 1167 (1998) arXiv:hep-th/9707088. D. A. Eliezer and R. P. Woodard, "Instability Of Higher Difference Initial Value Theories," Phys. Rev. D 40, 465 (1989). T. C. Cheng, P. M. Ho and M. C. Yeh, "Perturbative approach to higher derivative and nonlocal theories," Nucl. Phys. B 625, 151 (2002) arXiv:hep-th/0111160. K. Bering, "A note on nonlocality and Ostrogradski's construction," arXiv:hep-th/0007192. T. Nakamura and S. Hamamoto, "Higher Derivatives and Canonical Formalisms," Prog. Theor. Phys. 95, 469 (1996) arXiv:hep-th/9511219. H. J. Schmidt, Phys. Rev. D 49, 6354 (1994) [Erratum-ibid. D 54, 7906 (1996)] arXiv:gr-qc/9404038]. F. Muller-Hoissen, "Higher Derivative Versus Second Order Field Equations," Annalen Phys. 48, 543 (1991). J. Z. Simon, "Higher Derivative Lagrangians, Nonlocality, Problems And Solutions," Phys. Rev. D 41, 3720 (1990). M. Ostogradski, Mem. Ac. St. Petersbourg VI 4385 (1850).

[2] I. A. Batalin and G. A. Vilkovisky, "Quantization Of Gauge Theories With Linearly Dependent Generators," Phys. Rev. D 28, 2567 (1983) [Erratum-ibid. D 30, 508 (1984)]. A. Schwarz, "Geometry of Batalin-Vilkovisky quantization," Commun. Math. Phys. 155, 249 (1993) arXiv:hep-th/9205088.

[3] C. B. Thorn, "String field theory", Phys. Rep. 174 (1989) 1.

[4] B. Zwiebach, "Closed string field theory: Quantum action and the B-V master equation," Nucl. Phys. B 390, 33 (1993) arXiv:hep-th/9206084. 
[5] W. Siegel, "Introduction To String Field Theory," Adv. Ser. Math. Phys. 8, 1 (1988).

[6] J. L. Barbon and E. Rabinovici, "Stringy fuzziness as the custodian of time-space noncommutativity," Phys. Lett. B 486, 202 (2000) arXiv:hep-th/0005073.

[7] L. Alvarez-Gaume, J. L. Barbon and R. Zwicky, "Remarks on time-space noncommutative field theories," JHEP 0105, 057 (2001) [arXiv:hep-th/0103069.

[8] M. Kaku and K. Kikkawa, "The Field Theory Of Relativistic Strings, Pt. 1: Trees," Phys. Rev. D 10, 1110 (1974). "The Field Theory Of Relativistic Strings. Pt. 2: Loops And Pomerons," Phys. Rev. D 10, 1823 (1974).

[9] A. Sen, "Rolling tachyon," JHEP 0204, 048 (2002) arXiv:hep-th/0203211.

[10] A. Sen, "Tachyon matter," arXiv:hep-th/0203265.

[11] A. Sen, "Field theory of tachyon matter," arXiv:hep-th/0204143.

[12] G. W. Gibbons, arXiv:hep-th/0204008. M. Fairbairn and M. H. Tytgat, arXiv:hepth/0204070. C. M. Chen, D. V. Gal'tsov and M. Gutperle, arXiv:hep-th/0204071. S. Mukohyama, arXiv:hep-th/0204084. A. Feinstein, arXiv:hep-th/0204140. T. Padmanabhan, arXiv:hep-th/0204150. A. Frolov, L. Kofman and A. A. Starobinsky, arXiv:hep-th/0204187. K. Hashimoto, arXiv:hep-th/0204203. D. Choudhury, D. Ghoshal, D. P. Jatkar and S. Panda, arXiv:hep-th/0204204. X. z. Li, J. g. Hao and D. j. Liu, arXiv:hep-th/0204252. G. Shiu and I. Wasserman, arXiv:hep-th/0205003. T. Padmanabhan and T. R. Choudhury, arXiv:hep-th/0205055. L. Kofman and A. Linde, arXiv:hep-th/0205121. H. B. Benaoum, arXiv:hep-th/0205140. M. Sami, arXiv:hep-th/0205146. M. Sami, P. Chingangbam and T. Qureshi, arXiv:hepth/0205179.

[13] S. Sugimoto and S. Terashima, "Tachyon matter in boundary string field theory," arXiv:hep-th/0205085.

[14] J. A. Minahan, "Rolling the tachyon in super BSFT," arXiv:hep-th/0205098.

[15] E. Witten, "Noncommutative Geometry And String Field Theory," Nucl. Phys. B 268, 253 (1986).

[16] L. Brekke, P. G. Freund, M. Olson and E. Witten, "Nonarchimedean String Dynamics," Nucl. Phys. B 302, 365 (1988).

[17] D. Ghoshal and A. Sen, Nucl. Phys. B 584, 300 (2000) arXiv:hep-th/0003278. 
[18] J. A. Minahan, "Quantum corrections in p-adic string theory," arXiv:hepth/0105312. "Mode interactions of the tachyon condensate in p-adic string theory," JHEP 0103, 028 (2001) arXiv:hep-th/0102071.

[19] J. A. Minahan and B. Zwiebach, "Field theory models for tachyon and gauge field string dynamics," JHEP 0009, 029 (2000) arXiv:hep-th/0008231.

[20] A. A. Gerasimov and S. L. Shatashvili, "On exact tachyon potential in open string field theory," JHEP 0010, 034 (2000) arXiv:hep-th/0009103.

[21] A. Sen, "Time evolution in open string field theory", hep-th/0207105.

[22] A. Sen, "Descent relations among bosonic D-branes," Int. J. Mod. Phys. A14, 4061 (1999) hep-th/9902105. "Stable non-BPS bound states of BPS D-branes," JHEP 9808, 010 (1998) [hep-th/9805019]. "Tachyon condensation on the brane antibrane system," JHEP 9808, 012 (1998) hep-th/9805170. "SO(32) spinors of type I and other solitons on brane-antibrane pair," JHEP 9809, 023 (1998) [hep-th/9808141.

[23] A. Sen and B. Zwiebach, "Large marginal deformations in string field theory", JHEP 0010, 009 (2000) hep-th/0007153

[24] D. Gaiotto, L. Rastelli, A. Sen and B. Zwiebach, "Ghost structure and closed strings in vacuum string field theory," hep-th/0111129. A. Hashimoto and N. Itzhaki, "Observables of string field theory," JHEP 0201, 028 (2002) arXiv:hep-th/0111092. G. Moore and W. Taylor, "The singular geometry of the sliver," JHEP 0201, 004 (2002) arXiv:hep-th/0111069. M. Kleban, A. E. Lawrence and S. H. Shenker, "Closed strings from nothing," Phys. Rev. D 64, 066002 (2001) arXiv:hepth/0012081]. A. Sen, "Fundamental strings in open string theory at the tachyonic vacuum," J. Math. Phys. 42, 2844 (2001) arXiv:hep-th/0010240. J. A. Harvey, P. Kraus, F. Larsen and E. J. Martinec, "D-branes and strings as non-commutative solitons," JHEP 0007, 042 (2000) arXiv:hep-th/0005031. G. W. Gibbons, K. Hori and P. Yi, "String fluid from unstable D-branes," Nucl. Phys. B 596, 136 (2001) arXiv:hep-th/0009061. O. Bergman, K. Hori and P. Yi, "Confinement on the brane," Nucl. Phys. B 580, 289 (2000) arXiv:hep-th/0002223.

[25] M. Gutperle and A. Strominger, "Spacelike branes," JHEP 0204, 018 (2002) arXiv:hep-th/0202210.

[26] B. Zwiebach, "Oriented open-closed string theory revisited," Annals Phys. 267, 193 (1998) arXiv:hep-th/9705241. 